\title{
Analysis of Reassignment Operators Used in Synchrosqueezing Transforms: With an Application to Instantaneous Frequency Estimation
}

\author{
Sylvain Meignen and Neha Singh
}

\begin{abstract}
In this paper, our goal is first to investigate the behavior of reassignment operators used in synchrosqueezing transforms applied to multicomponent signals made of the superposition of amplitude and frequency modulated modes. Indeed, while these operators are associated with instantaneous frequency estimators very accurate on specific types of modes, the quality of the former worsens drastically when the modes depart from the ideal case they are designed for. We show in this paper that this particularly true when the modes interfere in the time-frequency plane or when some noise is present. Based on that analysis, we propose a novel instantaneous frequency estimator that only makes use of some specific points located on the ridges of synchrosqueezing transforms, and compare its performance with state-of-the-art techniques based on the same type of time-frequency representations.
\end{abstract}

Index Terms-time-frequency analysis, Fourier-based synchrosqueezing transform, reassignment methods

\section{INTRODUCTION}

M ULTICOMPONENT signals (MCSs) are very often used to represent non-stationary signals encountered in many different fields such as pathology diagnosis [1], [2], structural damage [3], [4] or physiological signals [5]. To analyze this type of signals the short-time Fourier transform (STFT) is very often considered since, with that time-frequency representation (TFR), the modes making up a MCS are associated with specific regions around ridges in the time-frequency (TF) plane [6], which consist of instantaneous frequency (IF) estimators of the modes [7], [8]. In that context, the IF estimates correspond to some specific local maxima of the spectrogram along the frequency axis. Other IF estimators can be designed by considering the reassignment operator used in the synchrosqueezing transform (SST), which can be applied to STFT [9], [10], to obtain the so-called Fourier-based synchrosqueezing transform (FSST), or to the continuous wavelet transform [11], [12]. SST has been widely used in various domains of applications among which fault diagnosis [13], [14], analysis of seismic signals [15], medical data analysis [5], [16], [17] and characterization of voice jitter [18] to name a few.

Variants of FSST were proposed to take into account the different nature of the modes to be reassigned. Indeed, in

The authors are with the Jean Kuntzmann Laboratory, University Grenoble Alpes and CNRS 5225, Grenoble 38401, France (emails: nehairo.iitr@gmail.com, sylvain.meignen@univ-grenoble-alpes.fr). This work was supported in part by the University Grenoble Alpes under IRS Grant "AMUSETE" and the ANR ASCETE project with grant number ANR-19CE48-0001-01. the seminal work of [9], [10] FSST used an IF estimator proved to be very accurate only when the modes can be locally approximated by pure harmonic modes. This approach was then extended by considering local linear chirp approximation for the modes in [19], [20], and then by assuming the modes have higher degree polynomial phases [21].

Nevertheless, the quality of the IF estimators used in FSSTs strongly depends on how well the modes are separated in the TF plane and on whether some noise is present. In particular, these estimates are ill-defined when the modes are crossing in the $\mathrm{TF}$ plane and a separation condition is assumed on the modes. It is worth noting here that recent works have aimed at dealing with crossing modes by estimating the chirp rate and IF simultaneously [22]. Meanwhile, many different techniques have been developed to improve mode separation by considering the so-called adaptive STFT [23]-[26].

However, to adapt the window length in STFT as is done in adaptive STFT does not warranty perfect IF estimation in noisy situations, and windows with different lengths at each time are to be used to estimate the IF of the different modes [24]. When only one window is used, the optimal window length is often computed using the properties of the Rényi entropy [27], [28], which is proved to lead to optimal concentration measure in the case of a linear chirp [29].

Our goal in this paper is three-fold. The first objective is to introduce a novel matricial form for the derivation of IF estimators used in FSSTs. This is done in Section II, in which we also introduce the notations that are used throughout the paper. The second objective is to characterize the zeros of the reassignment vectors associated with different types of FSSTs, then to derive approximations of these reassignment vectors, as explained in Section III, to finally investigate the behaviors of the reassignment vectors in the case of interfering pure harmonic modes and of noisy linear chirps. This is carried out respectively in Sections IV and V. The last objective of this paper is to propose a new IF estimator based on the determination of relevant points extracted from FSSTs ridges. The motivation for the selection of these relevant points is detailed at the beginning of Section VI, and the presentation of the new IF estimator follows. Numerical results illustrating its performance compared with state-of-the-art techniques based on FSSTs on both simulated and real signals conclude the paper. 


\section{NOTATIONS}

\section{A. Short-Time Fourier Transform}

In this section, we introduce a series of definitions we use throughout the paper. Considering a signal $f \in L^{1}(\mathbb{R}) \cap L^{2}(\mathbb{R})$ and a real window $h \in L^{\infty}(\mathbb{R}) \cap L^{2}(\mathbb{R})$, the (modified) ShortTime Fourier Transform (STFT) is defined as:

$$
V_{f}^{h}(t, \eta)=\int_{\mathbb{R}} f(\tau) h(\tau-t) e^{-2 i \pi(\tau-t) \eta} d \tau .
$$

In the sequel, we are going to study multicomponent signals (MCSs), defined as the superposition of several AM-FM components as follows:

$$
f(t)=\sum_{k=1}^{P} A_{k}(t) e^{2 i \pi \phi_{k}(t)}
$$

in which we assume $A_{k}(t)$ is positive, and $\phi_{k}^{\prime}(t)>0$ such that $\phi_{k+1}^{\prime}(t)>\phi_{k}^{\prime}(t)$ where $\phi_{k}^{\prime}(t)$ denotes the instantaneous frequency (IF) of mode $f_{k}$ at time $t$.

\section{B. Instantaneous Frequency Estimators Used in Synchrosqueezing Transforms}

The IF of each mode of $f$ can be estimated from STFT by means of a so-called local instantaneous frequency (LIF) estimator, used in Fourier-based synchrosqueezing transform (FSST). First, one considers the complex estimate:

$$
\tilde{\omega}_{f}(t, \eta)=\frac{\partial_{t} V_{f}^{h}(t, \eta)}{2 i \pi V_{f}^{h}(t, \eta)}=\eta-\frac{1}{2 i \pi} \frac{V_{f}^{h^{\prime}}(t, \eta)}{V_{f}^{h}(t, \eta)},
$$

and then defines the LIF estimator as

$$
\widehat{\omega}_{f}(t, \eta)=\Re\left\{\tilde{\omega}_{f}(t, \eta)\right\}=\eta-\Re\left\{\frac{1}{2 i \pi} \frac{V_{f}^{h^{\prime}}(t, \eta)}{V_{f}^{h}(t, \eta)}\right\} .
$$

FSST then consists of reassigning STFT through:

$$
T_{f}^{h}(t, \omega)=\int_{\left|V_{f}^{h}(t, \eta)\right|>\gamma} V_{f}^{h}(t, \eta) \delta\left(\omega-\widehat{\omega}_{f}(t, \eta)\right) d \eta,
$$

where $\delta$ is the Dirac distribution and $\gamma$ some threshold.

The quality of LIF estimator $\widehat{\omega}_{f}$ is however only satisfactory when the signal is made of perturbed purely harmonic modes. To overcome this limitation, a second-order LIF estimator was introduced based on a local linear chirp approximation [19], [20] and then used in the definition of second-order Fourierbased synchrosqueezing transform (FSST2). More precisely, introducing the complex time delay:

$$
\tilde{t}_{f}(t, \eta)=t-\frac{\partial_{\eta} V_{f}^{h}(t, \eta)}{2 i \pi V_{f}^{h}(t, \eta)}=t+\frac{V_{f}^{t h}(t, \eta)}{V_{f}^{h}(t, \eta)},
$$

and then the complex frequency modulation operator [21] (we omit $(t, \eta)$ for the sake of simplicity):

$$
\tilde{q}_{f}=\frac{\partial_{\eta} \tilde{\omega}_{f}}{\partial_{\eta} \tilde{t}_{f}}=\frac{1}{2 i \pi} \frac{V_{f}^{h^{\prime}} V_{f}^{t h}-V_{f}^{h} V_{f}^{t h^{\prime}}-\left(V_{f}^{h}\right)^{2}}{V_{f}^{h} V_{f}^{t^{2} h}-\left(V_{f}^{t h}\right)^{2}},
$$

the second-order complex LIF estimator is defined by:

$$
\tilde{\omega}_{f}^{[2]}=\left\{\begin{array}{c}
\tilde{\omega}_{f}+\tilde{q}_{f} \times\left(t-\tilde{t}_{f}\right) \text { if } \partial_{\eta} \tilde{t}_{f} \neq 0 \\
\tilde{\omega}_{f} \text { otherwise, }
\end{array}\right.
$$

and $\widehat{\omega}_{f}^{[2]}=\Re\left\{\tilde{\omega}_{f}^{[2]}\right\}$ is the sought second-order LIF estimator. FSST2 is then defined by replacing $\widehat{\omega}_{f}$ by $\widehat{\omega}_{f}^{[2]}$ in (5).

New LIF estimators were then proposed to handle MCSs containing AM-FM modes having non-negligible $l$ th order phase derivative with $l \geq 3$, especially those with fast oscillating phase [21]. In a nutshell, let us consider the Gaussian modulated linear chirp such that $f(\tau)=A(\tau) e^{i 2 \pi \phi(\tau)}$ with $\log (A(\tau))($ resp. $\phi(\tau))$ a polynomial of order $S$ (resp. $N)$ for $\tau$ close to $t$, with $S \leq N$, namely:

$$
=\exp \left(\sum_{j=0}^{N} \frac{\left([\log (A)]^{(j)}(t)+i 2 \pi \phi^{(j)}(t)\right)(\tau-t)^{j}}{j !}\right) .
$$

From (9), and the definition of STFT we may write:

$$
\begin{array}{r}
\partial_{t} V_{f}^{h}(t, \eta)=\quad\left([\log (A)]^{\prime}(t)+2 i \pi \phi^{\prime}(t)\right) V_{f}^{h}(t, \eta) \\
+\sum_{j=2}^{N} r_{j}^{[N]}(t) V_{f}^{t^{j-1} h}(t, \eta) \\
=r_{1}^{[N]}(t) V_{f}^{h}(t, \eta)+\sum_{j=2}^{N} r_{j}^{[N]}(t) V_{f}^{t^{j-1} h}(t, \eta)
\end{array}
$$

where $r_{j}^{[N]}(t)=\frac{[\log (A)]^{(j)}(t)+2 i \pi \phi^{(j)}(t)}{(j-1) !}$.

Now, when $f$ is a multicomponent signal defined as in (2) and made of modes following (9), the equality (10) turns into an approximation, namely for $(t, \eta)$ in the vicinity of $\left(t, \phi_{k}^{\prime}(t)\right)$ for some $k$, one may write:

$$
\begin{array}{r}
\partial_{t} V_{f}^{h}(t, \eta) \\
{[N](t, \eta) V_{f}^{h}(t, \eta)+\sum_{j=2}^{N} r_{j}^{[N]}(t, \eta) V_{f}^{t^{j-1}} h(t, \eta),}
\end{array}
$$

where $r_{j}^{[N]}(t, \eta) \approx \frac{\left[\log \left(A_{k}\right)\right]^{(j)}(t)+2 i \pi \phi_{k}^{(j)}(t)}{(j-1) !}$. In that context, $\widehat{\omega}_{f}^{[N]}(t, \eta):=\Re\left\{\frac{r_{1}^{[N]}(t, \eta)}{2 i \pi}\right\}$ is the $N$ th order LIF estimator of $f_{k}$. A simple way to compute $r_{1}^{[N]}$ is to come back to Eq. (11) which, remarking that $\partial_{\eta} V_{f}^{h}(t, \eta)=-2 i \pi V_{f}^{t h}(t, \eta)$, can be written under the matrix form:

$$
\begin{gathered}
{\left[\begin{array}{c}
\partial_{t} V_{f}^{h} \\
\frac{i}{2 \pi} \partial_{\eta} \partial_{t} V_{f}^{h} \\
\vdots \\
\frac{i^{N-1}}{(2 \pi)^{N-1}} \partial_{\eta}^{N-1} \partial_{t} V_{f}^{h}
\end{array}\right]} \\
=\left[\begin{array}{cccc}
V_{f}^{h} & V_{f}^{t h} & \ldots & V_{f}^{t^{N-1} h} \\
V_{f}^{t h} & V_{f}^{t^{2} h} & \ldots & V_{f}^{t^{N} h} \\
\vdots & \vdots & \ddots & \vdots \\
V_{f}^{t^{N-1} h} & V_{f}^{t^{N} h} & \ldots & V_{f}^{t^{2(N-1)} h}
\end{array}\right]\left[\begin{array}{c}
r_{1}^{[N]} \\
r_{2}^{[N]} \\
\vdots \\
r_{N}^{[N]}
\end{array}\right]=D R .
\end{gathered}
$$

Based on simple properties of the determinant of matrices, 
one obtains that:

$$
r_{1}^{[N]}=\frac{\operatorname{det}\left(M_{1}\right)}{\operatorname{det}(D)}
$$

with

$$
M_{1}=\left[\begin{array}{cccc}
\partial_{t} V_{f}^{h} & V_{f}^{t h} & \cdots & V_{f}^{t^{N-1} h} \\
\frac{i}{2 \pi} \partial_{\eta} \partial_{t} V_{f}^{h} & V_{f}^{t^{2} h} & \cdots & V_{f}^{t^{N} h} \\
\vdots & \vdots & \ddots & \vdots \\
\frac{i^{N-1}}{(2 \pi)^{N-1}} \partial_{\eta}^{N-1} \partial_{t} V_{f}^{h} & V_{f}^{t^{N} h} & \cdots & V_{f}^{t^{2(N-1)} h}
\end{array}\right] .
$$

Then, as $\partial_{t} V_{f}^{h}=i 2 \pi \eta V_{f}^{h}-V_{f}^{h^{\prime}}$, one gets, for any $l \geq 1$ :

$$
\partial_{\eta}^{l} \partial_{t} V_{f}^{h}=(-2 i \pi)^{l}\left(-k V_{f}^{t^{l-1} h}-V_{f}^{t^{l} h^{\prime}}+2 i \pi \eta V_{f}^{t^{l} h}\right),
$$

leading to: $\operatorname{det}\left(M_{1}\right)=i 2 \pi \eta \operatorname{det}(D)-\operatorname{det}\left(U_{1}\right)-\operatorname{det}\left(V_{1}\right)$ with:

$$
\begin{array}{r}
U_{1}=\left[\begin{array}{cccc}
0 & V_{f}^{t h} & \cdots & V_{f}^{t^{N-1} h} \\
V_{f}^{h} & V_{f}^{t^{2} h} & \cdots & V_{f}^{t^{N} h} \\
\vdots & \vdots & \ddots & \vdots \\
(N-1) V_{f}^{t^{N-2} h} & V_{f}^{t^{N} h} & \cdots & V_{f}^{t^{2(N-1)} h}
\end{array}\right], \\
V_{1}=\left[\begin{array}{cccc}
V_{f}^{h^{\prime}} & V_{f}^{t h} & \cdots & V_{f}^{t^{N-1} h} \\
V_{f}^{t h^{\prime}} & V_{f}^{t^{2} h} & \cdots & V_{f}^{t^{N} h} \\
\vdots & \vdots & \ddots & \vdots \\
V_{f}^{t^{N-1}} h^{\prime} & V_{f}^{t^{N} h} & \cdots & V_{f}^{t^{2(N-1)} h}
\end{array}\right]
\end{array}
$$

and thus

$$
\widehat{\omega}_{f}^{[N]}=\frac{\Im\left\{r_{1}^{[N]}\right\}}{2 \pi}=\eta-\frac{1}{2 \pi} \Im\left\{\frac{\operatorname{det}\left(U_{1}\right)+\operatorname{det}\left(V_{1}\right)}{\operatorname{det}(D)}\right\} .
$$

\section{Simplified LIF Estimators when h is a Gaussian Window}

When $h$ is the Gaussian window $e^{-\pi \frac{t^{2}}{\sigma^{2}}}$, the LIF estimators introduced above have simpler expressions. Indeed, as $h^{\prime}(t)=$ $-\frac{2 \pi}{\sigma^{2}} t h(t)$, one has:

$$
\widehat{\omega}_{f}=\eta+\Im\left\{\frac{1}{\sigma^{2}} \frac{V_{f}^{t h}}{V_{f}^{h}}\right\},
$$

and we may rewrite $\widehat{\omega}_{f}^{[2]}$ as:

$$
\begin{array}{r}
\widehat{\omega}_{f}^{[2]}=\widehat{\omega}_{f}+\Re\left\{\frac{1}{2 i \pi} \frac{\left(V_{f}^{h}\right)^{2}+V_{f}^{h} V_{f}^{t h^{\prime}}-V_{f}^{h^{\prime}} V_{f}^{t h}}{V_{f}^{h} V_{f}^{t^{2} h}-\left(V_{f}^{t h}\right)^{2}} \frac{V_{f}^{t h}}{V_{f}^{h}}\right\} \\
=\widehat{\omega}_{f}+\Re\left\{\frac{1}{2 i \pi} \frac{V_{f}^{h} V_{f}^{t h}}{V_{f}^{h} V_{f}^{t^{2} h}-\left(V_{f}^{t h}\right)^{2}}\right\}-\Im\left\{\frac{1}{\sigma^{2}} \frac{V_{f}^{t h}}{V_{f}^{h}}\right\} \\
=\widehat{\omega}_{f}+\Re\left\{\frac{1}{2 i \pi} \frac{V_{f}^{h} V_{f}^{t h}}{V_{f}^{h} V_{f}^{t^{2} h}-\left(V_{f}^{t h}\right)^{2}}\right\}-\left(\widehat{\omega}_{f}-\eta\right) \\
=\eta+\Im\left\{\frac{1}{2 \pi} \frac{V_{f}^{h} V_{f}^{t h}}{V_{f}^{h} V_{f}^{t 2 h}-\left(V_{f}^{t h}\right)^{2}}\right\} .
\end{array}
$$

An alternative technique to compute a LIF estimator still assuming a local linear chirp approximation for the modes is to consider that the complex modulation operator is not defined by (7) but by [19]:

$$
\bar{q}_{f}=\frac{\partial_{t} \tilde{\omega}_{f}}{\partial_{t} \tilde{t}_{f}}=\frac{1}{2 i \pi} \frac{V_{f}^{h^{\prime \prime}} V_{f}^{h}-\left(V_{f}^{h^{\prime}}\right)^{2}}{V_{f}^{t h} V_{f}^{h^{\prime}}-V_{f}^{t h^{\prime}} V_{f}^{h}} .
$$

Replacing $\tilde{q}_{f}$ by $\bar{q}_{f}$ in (8), one obtains a new LIF estimator, which is actually the same as the one introduced in [21]. Indeed, one has

$$
\begin{array}{r}
\frac{1}{2 i \pi} \frac{V_{f}^{h^{\prime \prime}} V_{f}^{h}-\left(V_{f}^{h^{\prime}}\right)^{2}}{V_{f}^{t h} V_{f}^{h^{\prime}}-V_{f}^{t h^{\prime}} V_{f}^{h}} \\
=\frac{1}{2 i \pi} \frac{\left(-V_{f}^{h}+\frac{2 \pi}{\sigma^{2}} V_{f}^{t^{2} h}\right) V_{f}^{h}-\frac{2 \pi}{\sigma^{2}}\left(V_{f}^{t h}\right)^{2}}{-\left(V_{f}^{t h}\right)^{2}+V_{f}^{t^{2} h} V_{f}^{h}} \\
=\quad \frac{1}{i \sigma^{2}}-\frac{\left(V_{f}^{h}\right)^{2}}{2 i \pi\left(V_{f}^{t^{2} h} V_{f}^{h}-\left(V_{f}^{t h}\right)^{2}\right)},
\end{array}
$$

and the LIF estimator obtained using $\bar{q}_{f}$ instead of $\tilde{q}_{f}$ reads:

$$
\begin{array}{r}
=\widehat{\omega}_{f}+\Re\left\{\left(-\frac{1}{i \sigma^{2}}+\frac{\left(V_{f}^{h}\right)^{2}}{2 i \pi\left(V_{f}^{t^{2} h} V_{f}^{h}-\left(V_{f}^{t h}\right)^{2}\right)}\right) \frac{V_{f}^{t h}}{V_{f}^{h}}\right\} \\
=\widehat{\omega}_{f}+\Re\left\{\frac{V_{f}^{h} V_{f}^{t h}}{2 i \pi\left(V_{f}^{t^{2} h} V_{f}^{h}-\left(V_{f}^{t h}\right)^{2}\right)}\right\}-\Im\left\{\frac{1}{\sigma^{2}} \frac{V_{f}^{t h}}{V_{f}^{h}}\right\} \\
=\widehat{\omega}_{f}^{[2]} .
\end{array}
$$

As far as higher order LIF estimators, i.e. $N>2$, are concerned, to use the Gaussian window also brings interesting simplifications. Indeed, in such a case, the first two columns of matrix $V_{1}$ introduced in (16) are colinear and thus its determinant is null. In that context, one may thus write:

$$
\widehat{\omega}_{f}^{[N]}=\frac{1}{2 \pi} \Im\left\{r_{1}^{[N]}\right\}=\eta-\frac{1}{2 \pi} \Im\left\{\frac{\operatorname{det}\left(U_{1}\right)}{\operatorname{det}(D)}\right\} .
$$

In the following sections, we will stick to the use of Gaussian windows since the studied LIF estimators have simpler forms. Also, as shown in the following section, to use Gaussian windows enables us to give very simple characterizations of the zeros of reassignment vectors and then to mathematically study the latter in the vicinity of their zeros.

\section{ON APPROXIMATIONS OF REASSIGNMENT VeCTORS IN THE VICINITY OF THEIR ZEROS}

In this section, we first investigate more in detail the nature of the zeros of the reassignment vectors namely of $\widehat{\omega}_{f}^{[N]}(t, \eta)-\eta$, focusing in particular on the cases $N=1$ and $N=2$. Then we propose different simple approximations of reassignment vectors in the vicinity of their zeros that will help us understand the differences between reassignment processes.

\section{A. Characterization of the Zeros of Reassignment Vectors}

To study the zeros of the reassignment vector, when $N=$ 1 , we first recall that (omitting again $(t, \eta)$ for the sake of 
simplicity):

$$
\begin{aligned}
\widehat{\omega}_{f}-\eta & =\Im\left\{\frac{1}{\sigma^{2}} \frac{V_{f}^{t h}}{V_{f}^{h}}\right\} \\
& =-\Im\left\{\frac{\partial_{\eta} V_{f}^{h}}{2 i \pi \sigma^{2} V_{f}^{h}}\right\}=\frac{1}{4 \pi \sigma^{2}} \frac{\partial_{\eta}\left|V_{f}^{h}\right|^{2}}{\left|V_{f}^{h}\right|^{2}},
\end{aligned}
$$

whose zeros correspond to the points $(t, \eta)$ such that $\partial_{\eta}\left|V_{f}^{h}(t, \eta)\right|^{2}=0$.

Now, we investigate the zeros of $\widehat{\omega}_{f}^{[2]}-\eta$ corresponding to:

$$
\widehat{\omega}_{f}^{[2]}-\eta=\Im\left\{\frac{1}{2 \pi} \frac{V_{f}^{h} V_{f}^{t h}}{V_{f}^{h} V_{f}^{t^{2} h}-\left(V_{f}^{t h}\right)^{2}}\right\}
$$

that is to say

$$
\begin{array}{r}
0=\Im\left\{V_{f}^{h} V_{f}^{t h}\left(V_{f}^{h} V_{f}^{t^{2} h}-\left(V_{f}^{t h}\right)^{2}\right)^{*}\right\} \\
=\left|V_{f}^{h}\right|^{2} \Im\left\{V_{f}^{t h}\left(V_{f}^{t^{2} h}\right)^{*}\right\}-\left|V_{f}^{t h}\right|^{2} \Im\left\{V_{f}^{h}\left(V_{f}^{t h}\right)^{*}\right\} \\
=\left|V_{f}^{h}\right|^{2} \Re\left\{V_{f}^{t h} \partial_{\eta}\left(V_{f}^{t h}\right)^{*}\right\}-\left|V_{f}^{t h}\right|^{2} \Re\left\{V_{f}^{h} \partial_{\eta}\left(V_{f}^{h}\right)^{*}\right\} \\
=\left|V_{f}^{h}\right|^{2} \partial_{\eta}\left|V_{f}^{t h}\right|^{2}-\left|V_{f}^{t h}\right|^{2} \partial_{\eta}\left|V_{f}^{h}\right|^{2} .
\end{array}
$$

which can also be viewed as

$$
\operatorname{det}\left[\begin{array}{cc}
\left|V_{f}^{h}\right|^{2} & \partial_{\eta}\left|V_{f}^{h}\right|^{2} \\
\left|V_{f}^{t h}\right|^{2} & \partial_{\eta}\left|V_{f}^{t h}\right|^{2}
\end{array}\right]=0
$$

The reassignment vector when $N=2$ thus reads:

$$
\widehat{\omega}_{f}^{[2]}-\eta=\frac{\left|V_{f}^{h}\right|^{2} \partial_{\eta}\left|V_{f}^{t h}\right|^{2}-\left|V_{f}^{t h}\right|^{2} \partial_{\eta}\left|V_{f}^{h}\right|^{2}}{\left|V_{f}^{h} V_{f}^{t^{2} h}-\left(V_{f}^{t h}\right)^{2}\right|^{2}} .
$$

To extend (26) to higher order $N$ is possible by considering the points $(t, \eta)$ such that $\Im\left\{\operatorname{det}\left(U_{1}\right) \operatorname{det}(D)^{*}\right\}=0$, but this leads to much more complex expressions, whose analysis is left for future work.

\section{B. Approximating Reassignment Vectors in the Vicinity of their Zeros}

We are now interested in approximating the different reassignment vectors in the vicinity of their zeros. We first approximate second order reassignment vector, i.e. $N=2$, considering that, in the vicinity of its zeros, $V_{f}^{t h}$ is small. Indeed, let us remark that the STFT of a linear chirp $f(t)=$ $A e^{2 i \pi \phi(t)}$ computed with the Gaussian window $g(t)=e^{-\pi \frac{t^{2}}{\sigma^{2}}}$ reads [19]:

$$
V_{f}^{h}(t, \eta)=V_{f}^{h}\left(t, \phi^{\prime}(t)\right) e^{\frac{-\pi \sigma^{2}\left(1+i \phi^{\prime \prime}(t) \sigma^{2}\right)}{1+\left(\phi^{\prime \prime}(t) \sigma^{2}\right)^{2}}\left(\eta-\phi^{\prime}(t)\right)^{2}} .
$$

Then from (28), we immediately get that $V_{f}^{t h}\left(t, \phi^{\prime}(t)\right)=$ $\frac{i}{2 \pi} \partial_{\eta} V_{f}^{h}\left(t, \phi^{\prime}(t)\right)=0$ and more generally that:

$$
\begin{array}{r}
V_{f}^{t^{l} h}(t, \eta)=\left(\frac{i}{2 \pi}\right)^{l} \partial_{\eta}^{l} V_{f}^{h}(t, \eta)=\frac{-\sigma^{2}\left(1+i \phi^{\prime \prime}(t) \sigma^{2}\right)}{1+\left(\phi^{\prime \prime}(t) \sigma^{2}\right)^{2}} \\
{\left[i\left(\eta-\phi^{\prime}(t)\right) V_{f}^{t^{l-1} h}(t, \eta)-\frac{l-1}{2 \pi} V_{f}^{t^{l-2} h}(t, \eta)\right],}
\end{array}
$$

implying that $V_{f}^{t^{l} h}\left(t, \phi^{\prime}(t)\right)$ is null when $l$ is odd. Based on this analysis, it is natural to consider the following approximation of $\widehat{\omega}_{f}^{[2]}(t, \eta)-\eta$ in the vicinity of its zeros:

$$
\begin{array}{r}
\widehat{\omega}_{f}^{[2]}(t, \eta)-\eta=\Im\left\{\frac{1}{2 \pi} \frac{V_{f}^{h} V_{f}^{t h}}{V_{f}^{h} V_{f}^{t^{2} h}-\left(V_{f}^{t h}\right)^{2}}\right\} \\
=\Im\left\{\frac{1}{2 \pi} \frac{V_{f}^{t h}}{V_{f}^{t^{2} h}} \frac{1}{1-\frac{\left(V_{f}^{t h}\right)^{2}}{V_{f}^{h} V_{f}^{2^{2}}}}\right\} \\
\approx \Im\left\{\frac{1}{2 \pi} \frac{V_{f}^{t h}}{V_{f}^{t^{2} h}}\right\}+\Im\left\{\frac{1}{2 \pi} \frac{\left(V_{f}^{t h}\right)^{3}}{V_{f}^{h}\left(V_{f}^{t^{2} h}\right)^{2}}\right\} .
\end{array}
$$

When $\frac{\left(V_{f}^{t h}\right)^{2}}{V_{f}^{h} V_{f}^{t^{2} h}}<<1$, one can approximate $\widehat{\omega}_{f}^{[2]}$ only including the first order term in $V_{f}^{t h}$ in approximation (30), which we denote by $\widehat{\omega}_{f, 1}^{[2]}$. In the opposite case, one should consider two terms in the approximation, namely third order terms in $V_{f}^{t h}$. We denote by $\widehat{\omega}_{f, 2}^{[2]}$ this approximation.

From now on, we call second order zeros the zeros of $\widehat{\omega}_{f}^{[2]}-$ $\eta$, and we define approximations of $\widehat{\omega}_{f}^{[3]}$ in the vicinity of these points when $f$ is a monocomponent satisfying (9) with $S \leq 2$. For that purpose, we first rewrite $\widehat{\omega}_{f}^{[3]}$ as a perturbation of $\widehat{\omega}_{f}^{[2]}$, namely:

Proposition III.1. Assume $f$ satisfies (9) with $\log (A)$ a second order polynomial, then:

$$
\widehat{\omega}_{f}^{[3]}=\widehat{\omega}_{f}^{[2]}+\Im\left\{\frac{r_{3}^{[3]}}{2 \pi}\right\} \Re\left\{\frac{\left(V_{f}^{t^{2} h}\right)^{2}-V_{f}^{t^{3} h} V_{f}^{t h}}{\left(V_{f}^{t h}\right)^{2}-V_{f}^{t^{2} h} V_{f}^{h}}\right\} .
$$

The proof is available in Appendix A. Assuming that both $V_{f}^{t h}$ and $V_{f}^{t^{3} h}$ are small close to the second order zeros, we get the following approximation of $\widehat{\omega}_{f}^{[3]}$ close to these points:

$$
\widehat{\omega}_{f, 1}^{[3]}=\widehat{\omega}_{f}^{[2]}+\Im\left\{\frac{r_{3}^{[3]}}{2 \pi}\right\} \Re\left\{\frac{-V_{f}^{t^{2} h}}{V_{f}^{h}}\right\} .
$$

The quality of this estimate will be investigated later in the paper.

\section{Analysis of Reassignment Vectors on INTERFERING PURE HARMONIC MODES}

In this section we study the behavior of reassignment vectors when the signal is made of interfering pure harmonics. First, we investigate the zeros of the reassignment vectors when $N=1$ or $N=2$, and the behavior of the latter in the vicinity of spectrogram ridges, corresponding to the points $\partial_{\eta}\left|V_{f}^{h}(t, \eta)\right|^{2}=0$. We then investigate the behavior of the third order reassignment vector in that context.

Let us consider that $f(t)=f_{1}(t)+f_{2}(t)$ with $f_{1}(t)=$ $A e^{i 2 \pi \xi_{1} t}$ and $f_{2}(t)=e^{i 2 \pi \xi_{2} t}$, where $\xi_{1}<\xi_{2}$. For such a signal and when $h$ is the Gaussian window mentioned above, one has $V_{f_{1}}^{h}(t, \eta)=\hat{h}\left(\eta-\xi_{1}\right) A e^{i 2 \pi \xi_{1} t}=\sigma A e^{i 2 \pi \xi_{1} t} e^{-\pi\left(\eta-\xi_{1}\right)^{2} \sigma^{2}}$ and $V_{f_{2}}^{h}(t, \eta)=\sigma e^{i 2 \pi \xi_{2} t} e^{-\pi\left(\eta-\xi_{2}\right)^{2} \sigma^{2}}$, and the spectrogram reads:

$$
\begin{aligned}
& \left|V_{f}^{h}(t, \eta)\right|^{2}=\sigma^{2}\left(A^{2} e^{-2 \pi \sigma^{2}\left(\eta-\xi_{1}\right)^{2}}+e^{-2 \pi \sigma^{2}\left(\eta-\xi_{2}\right)^{2}}\right. \\
& \left.+2 A e^{-\pi \sigma^{2}\left[\left(\eta-\xi_{1}\right)^{2}+\left(\eta-\xi_{2}\right)^{2}\right]} \cos \left(2 \pi\left(\xi_{2}-\xi_{1}\right) t\right)\right)
\end{aligned}
$$




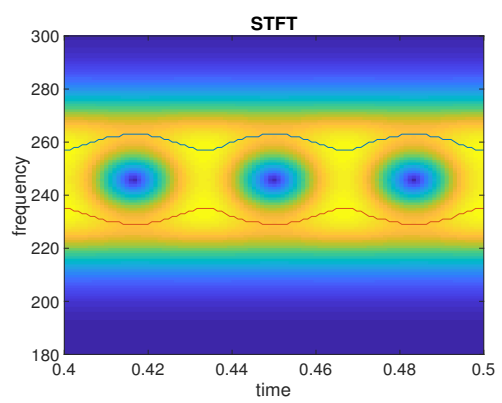

(a)

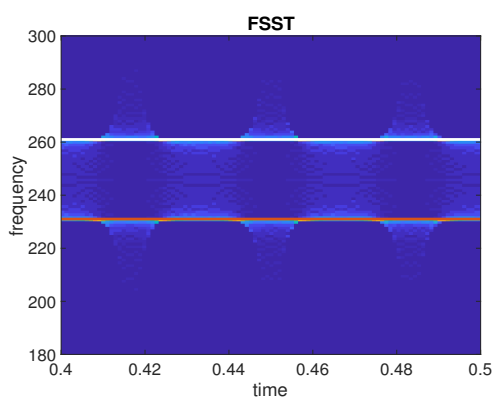

(b)

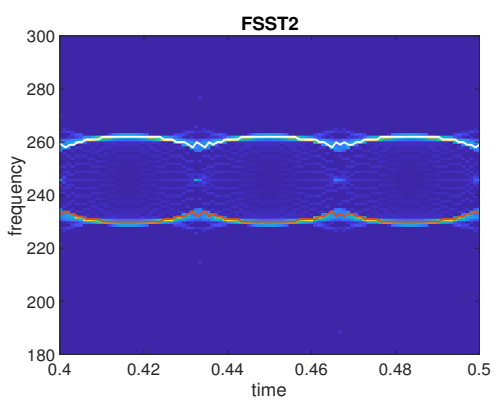

(c)

Fig. 1: (a): STFT of two interfering modes, with the two ridges associated with local maxima superimposed; (b): FSST of the signal in (a); (c): FSST2 of the signal in (a)

\section{A. Determination of the Zeros Common to First and Second Order Reassignment Vectors}

We here investigate the set of TF points consisting of the zeros common to the first and second order reassignment vector. In the previous section, we showed that the zeros at time $t$ of $\widehat{\omega}_{f}-\eta$ corresponded to $\eta$ satisfying $\partial_{\eta}\left|V_{f}^{h}(t, \eta)\right|^{2}=0$ and $V_{f}^{h}(t, \eta) \neq 0$. We can then prove the following result:

Proposition IV.1. A zero $(t, \eta)$ of $\widehat{\omega}_{f}-\eta$, such that $V_{f}^{h} V_{f}^{t^{2} h}-$ $\left(V_{f}^{t h}\right)^{2} \neq 0$ is a zero of $\widehat{\omega}_{f}^{[2]}-\eta$ if and only if

$$
A\left(\eta-\xi_{1}\right) e^{-\pi \sigma^{2}\left(\eta-\xi_{1}\right)^{2}}= \pm\left(\eta-\xi_{2}\right) e^{-\pi \sigma^{2}\left(\eta-\xi_{2}\right)^{2}}
$$

The proof is available in Appendix B. The time instants associated with these points then obey the following rule:

Proposition IV.2. If $(t, \eta)$ is a zero common to $\widehat{\omega}_{f}-\eta$ and $\widehat{\omega}_{f}^{[2]}-\eta$ such that $A\left(\eta-\xi_{1}\right) e^{-\pi \sigma^{2}\left(\eta-\xi_{1}\right)^{2}}=-(\eta-$ $\left.\xi_{2}\right) e^{-\pi \sigma^{2}\left(\eta-\xi_{2}\right)^{2}} \quad$ (resp. $A\left(\eta-\xi_{1}\right) e^{-\pi \sigma^{2}\left(\eta-\xi_{1}\right)^{2}}=(\eta-$ $\left.\xi_{2}\right) e^{-\pi \sigma^{2}\left(\eta-\xi_{2}\right)^{2}}$ ), then $t=t_{k}=\frac{k}{\xi_{2}-\xi_{1}}$ for some $k \in \mathbb{Z}$ (resp. $\tilde{t}_{k}=\frac{k+1 / 2}{\xi_{2}-\xi_{1}}$ for some $k \in \mathbb{Z}$ ).

The proof is available in Appendix C. Note that the time instants $t_{k}$ (resp. $\tilde{t}_{k}$ ) when $k$ varies are associated with local maxima (resp. minima) of $\left|V_{f}^{h}(., \eta)\right|^{2}$. Furthermore, we can also remark that the locations $t_{k}$ (resp. $\tilde{t}_{k}$ ) correspond to local maxima (resp. minima) of the spectrogram along the spectrogram ridges.

\section{B. Analysis of Second Order Reassignment Vector in the Vicinity of Spectrogram Ridges}

Let us first remark that at location $t_{k}$ and $\tilde{t}_{k}$ defined in the previous section we have for any $\eta$ :

$$
\begin{aligned}
\left|V_{f}^{h}\left(t_{k}, \eta\right)\right|^{2} & =\left(A e^{-\pi \sigma^{2}\left(\eta-\xi_{1}\right)^{2}}+e^{-\pi \sigma^{2}\left(\eta-\xi_{2}\right)^{2}}\right)^{2} \\
\left|V_{f}^{h}\left(\tilde{t}_{k}, \eta\right)\right|^{2} & =\left(A e^{-\pi \sigma^{2}\left(\eta-\xi_{1}\right)^{2}}-e^{-\pi \sigma^{2}\left(\eta-\xi_{2}\right)^{2}}\right)^{2} .
\end{aligned}
$$

At time $t_{k}$, when the level of interference is not too high $\left|V_{f}^{h}\left(t_{k}, .\right)\right|^{2}$ has three extrema, located respectively at $\eta_{0}^{\max }<\eta_{1}^{\max }<\eta_{2}^{\max }$. We use the superscript max to recall that $\left|V_{f}^{h}(., \eta)\right|^{2}$ is maximum at $t_{k}$. Note that $\eta_{0}^{\max }$ and $\eta_{2}^{\max }$ correspond to maxima and $\eta_{1}^{\max }$ to a minimum of $\left|V_{f}^{h}\left(t_{k}, .\right)\right|^{2}$. Similarly, at time $\tilde{t}_{k},\left|V_{f}^{h}\left(\tilde{t}_{k}, .\right)\right|^{2}$ has three extrema located at $\eta_{0}^{\min }<\eta_{1}^{\min }<\eta_{2}^{\min }$ (the superscript min recalling that $\left|V_{f}^{h}(., \eta)\right|^{2}$ is minimum at $\left.\tilde{t}_{k}\right)$. Note that $\eta_{0}^{\min }$ and $\eta_{2}^{\min }$ correspond to maxima and $\eta_{1}^{\min }$ to a minimum of $\left|V_{f}^{h}\left(\tilde{t}_{k}, .\right)\right|^{2}$. Finally, one can also remark that the point $\left(\tilde{t}_{k}, \eta_{1}^{\min }\right)$ is a zero of the spectogram.

The points $\left(t_{k}, \eta_{2}^{\max }\right)$ and $\left(\tilde{t}_{k}, \eta_{2}^{\min }\right)$ are part of what we call from now on the upper spectrogram ridge (see the blue curve in Fig. 1 (a)), while $\left(t_{k}, \eta_{0}^{\max }\right)$ and $\left(\tilde{t}_{k}, \eta_{0}^{\min }\right)$ belong to the lower spectrogram ridge (see the red curve in Fig. 1 (a)). Investigating the behavior of the second order reassignment vector on the upper and lower spectrogram ridges, one founds the following property:

Proposition IV.3. On the upper (resp. lower) spectrogram ridge the second order reassignment vector is oriented towards higher (resp. lower) frequencies except at time instants $t_{k}$ and $\tilde{t}_{k}$.

The proof is available in Appendix D. An illustration of Proposition IV.3 is given in Fig. 1 (c), in which we see that, by comparing with Fig. 1 (a), the TF coefficients are not reassigned onto the spectrogram ridges with FSST2: the point on the upper (resp. lower) spectrogram ridge (except those at time $t_{k}$ and $\tilde{t}_{k}$ ) are reassigned at a higher (resp. lower) frequency. Finally, note that, even if the spectrogram ridges are the zeros of the first order reassignment vector, this does not mean FSST ridges, i.e. TF curves corresponding to local maxima of the modulus of FSST along the frequency axis, are the same as the spectrogram ridges (see Fig. 1 (b)). Indeed, from Fig. 1 (b), FSST ridges appear to be accurate estimates of the IF of the modes which is not the case of the spectrogram ridges.

To explain why FSST ridges lead to better IF estimates than FSST2 ridges, we investigate the behavior of the different reassignment operators $\widehat{\omega}_{f}$ and $\widehat{\omega}_{f}^{[2]}$ in the vicinity of the lower spectrogram ridge, at location $t_{k}$ and $\tilde{t}_{k}$, in Fig. 2 (a) and (b), respectively. We notice from Fig. 2 (a) that, at location $t_{k}$, all the STFT coefficients below this spectrogram ridge are all reassigned with $\widehat{\omega}_{f}$ to a TF location closer to the true IF than when $\widehat{\omega}_{f}^{[2]}$ is used. From Fig. 2 (b), a similar conclusion can be drawn at time $\tilde{t}_{k}$. On the contrary, both reassignment vectors are very inaccurate between the spectrogram ridges.

Also to better understand the behavior of $\widehat{\omega}_{f}^{[2]}$ in the vicinity 


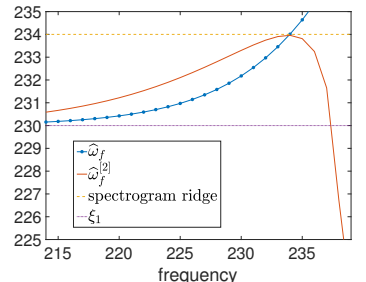

(a)

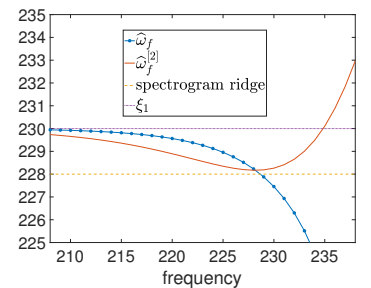

(b)

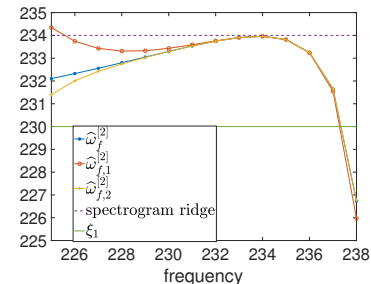

(c)

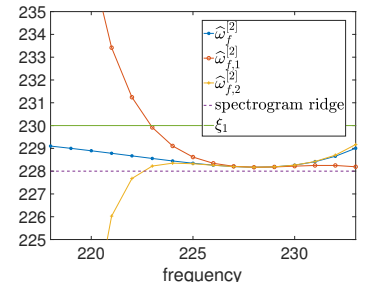

(d)

Fig. 2: (a): $\widehat{\omega}_{f}$ and $\widehat{\omega}_{f}^{[2]}$ in the vicinity of the lower spectrogram ridge at time $t_{k}$; (b): same as (a) but at time $\tilde{t}_{k}$; (c): $\widehat{\omega}_{f}^{[2]}$, $\widehat{\omega}_{f, 1}^{[2]}$, and $\widehat{\omega}_{f, 2}^{[2]}$ in the vicinity of the lower spectrogram ridge at time $t_{k} ;(\mathrm{d})$ : same as (c) but at time $\tilde{t}_{k}$.

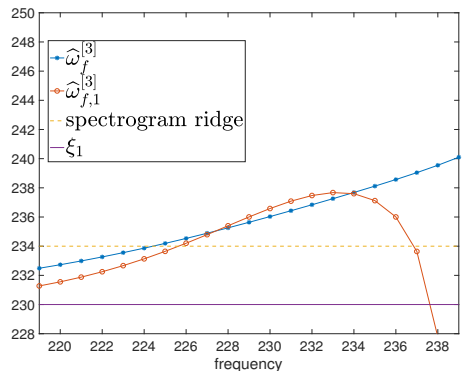

(a)

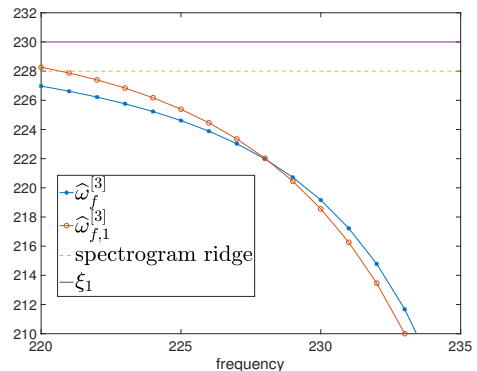

(b)

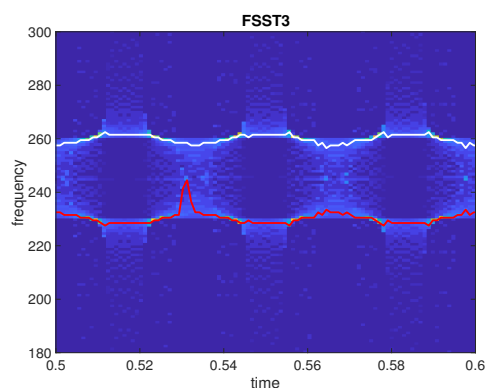

(c)

Fig. 3: (a): $\widehat{\omega}_{f}^{[3]}, \widehat{\omega}_{f, 1}^{[3]}$ around TF point $\left(t_{k}, \eta_{1}^{\max }\right)$, when $\eta$ varies along with the location of the spectrogram at $t_{k}$ and $\xi_{1}$; (b): same as (a) but around point $\left(\tilde{t}_{k}, \eta_{1}^{\min }\right)$; (c): modulus of FSST3 for the interfering modes along with the corresponding ridges.

of the lower spectrogram ridge, we plot in Fig. 2 (c) and (d) its approximations $\widehat{\omega}_{f, 1}^{[2]}$ and $\widehat{\omega}_{f, 2}^{[2]}$, defined right after (30), at time $t_{k}$ and $\tilde{t}_{k}$. While close to the ridge these approximations are correct, the hypothesis that $V_{f}^{t h}$ is small becomes rapidly erroneous as one moves away from the lower spectrogram ridge towards lower frequencies, and the proposed simple approximations of $\widehat{\omega}_{f}^{[2]}$ are no longer valid.

\section{Behavior of Third Order Synchrosqueezing Transform in the Vicinity of Spectogram Ridges}

Let us now consider a point below and close to the lower spectrogram ridge at time $\tilde{t}_{k}$. Denote by $\left(t, \psi_{0}^{\prime}(t)\right)$ the spectrogram ridge in the vicinity of that point. We first remark that $\Im\left\{\frac{r_{3}^{[3]}\left(t, \psi_{0}^{\prime}(t)\right)}{2 \pi}\right\} \approx \psi_{0}^{(3)}(t)$ which is such that $\psi_{0}^{(3)}\left(\tilde{t}_{k}\right)>0$ (see Fig. 1 (a), lower ridge). Then making a pure harmonic approximation, we get that $\Re\left\{\frac{-V_{f}^{t^{2} h}}{V_{f}^{h}}\right\} \approx-\frac{\sigma^{2}}{2 \pi}<0$ at these points. From this, we deduce that $\widehat{\omega}_{f, 1}^{[3]}$ is smaller than $\widehat{\omega}_{f}^{[2]}$ at these points on the lower spectrogram ridge. As $\widehat{\omega}_{f}^{[2]}$ underestimates the IF of the mode (see Fig. 2 (b)), $\widehat{\omega}_{f, 1}^{[3]}$ underestimates even more that IF. Now as $\widehat{\omega}_{f, 1}^{[3]}$ is a good approximation of $\widehat{\omega}_{f}^{[3]}$ in the vicinity of spectrogram ridges (see Fig. 3 (b)), such is also the case when one uses $\widehat{\omega}_{f}^{[3]}$ : the reassigned value when using $\widehat{\omega}_{f}^{[3]}$ are farther from $\xi_{1}$ than the one given by $\widehat{\omega}_{f}$ or $\widehat{\omega}_{f}^{[2]}$. Similarly, at time $t_{k}$, considering a point below and close to the lower spectrogram ridge, since $\psi_{0}^{(3)}\left(t_{k}\right)<0$ and using the same pure harmonic approximation as previously, $\widehat{\omega}_{f, 1}^{[3]}$ is larger than $\widehat{\omega}_{f}^{[2]}$. As $\widehat{\omega}_{f}^{[2]}$ overestimates the IF of the mode at this point (see Fig. 2 (a)), this overestimation will be even larger at this point with $\widehat{\omega}_{f, 1}^{[3]}$. Remarking $\widehat{\omega}_{f, 1}^{[3]}$ is a good approximation of $\widehat{\omega}_{f}^{[3]}$ (see Fig. 3 (a)), this is also the case with $\widehat{\omega}_{f}^{[3]}$. The modulus of FSST3 for the signal of Fig. 1 (a) along with its ridges are finally displayed in Fig. 4 (c), and we notice that, as expected, these are less accurate IF estimates than FSST or FSST2 ridges.

\section{Analysis of Third Order Reassignment Vectors ON NOISY LINEAR CHIRPS}

In this section, our goal is to explain why LIF estimators associated with third order reassignment vectors is worse than that with $N=2$ when applied to noisy linear chirps.

Let us consider that $f(t)=A(t) e^{2 i \pi \phi(t)}$ is a Gaussian modulated linear chirp (i.e. $\log (A)$ is a polynomial of order 2 at most, and $\phi^{\prime \prime}(t)=C$ ). Adding some complex Gaussian white noise $n$ to $f$ one obtains $\tilde{f}=f+n$. We now analyze the behavior of $\widehat{\omega}_{\tilde{f}, 1}^{[3]}$ in the vicinity of the spectrogram ridge associated with the noisy linear chirp and denoted by $\left(t, \phi_{n}^{\prime}(t)\right)$, the subscript meaning some noise is present. Assuming the hypotheses of Proposition III.1 hold, and from (32), we may write:

$$
\widehat{\omega}_{\tilde{f}, 1}^{[3]}-\widehat{\omega}_{\tilde{f}}^{[2]} \approx \Im\left\{\frac{r_{3}^{[3]}}{2 \pi}\right\} \Re\left\{-\frac{V_{\tilde{f}}^{t^{2} h}}{V_{\tilde{f}}^{h}}\right\} .
$$


As from (29) we have that

$$
\Re\left\{-\frac{V_{f}^{t^{2} h}\left(t, \phi^{\prime}(t)\right)}{V_{f}^{h}\left(t, \phi^{\prime}(t)\right)}\right\}=-\frac{1}{1+\left(\phi^{\prime \prime}(t) \sigma^{2}\right)^{2}} \frac{\sigma^{2}}{2 \pi},
$$

$\Re\left\{-\frac{V_{\tilde{f}}^{t^{2} h}}{V_{\tilde{f}}^{h}}\right\}$ remains negative in the vicinity of $\left(t, \phi_{n}^{\prime}(t)\right)$ when the noise level is reasonable. Thus, recalling that $\Im\left\{\frac{r_{3}^{[3]}\left(t, \phi_{n}^{\prime}(t)\right)}{2 \pi}\right\} \approx \phi_{n}^{(3)}(t), \widehat{\omega}_{\tilde{f}, 1}^{[3]}\left(t, \phi_{n}^{\prime}(t)\right)-\widehat{\omega}_{\tilde{f}}^{[2]}\left(t, \phi_{n}^{\prime}(t)\right)$ is positive when $\phi_{n}^{\prime}(t)$ is concave and negative otherwise. Finally, we numerically notice that $\widehat{\omega}_{\tilde{f}, 1}^{[3]}$ still behaves very similarly to $\widehat{\omega}_{\tilde{f}}^{[3]}$ in the vicinity of the spectrogram ridge corresponding to the noisy linear chirp. So we can conclude that if the ridge $\left(t, \phi_{n}^{\prime}(t)\right)$ is oscillating then the oscillations will be more important on FSST3 ridge than on FSST2 ridge. To confirm this, we consider a noisy linear chirp whose FSST3

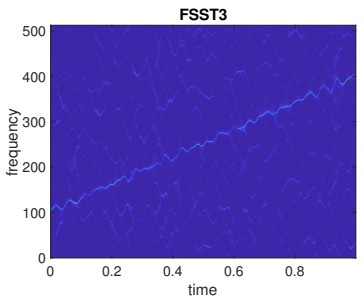

(a)

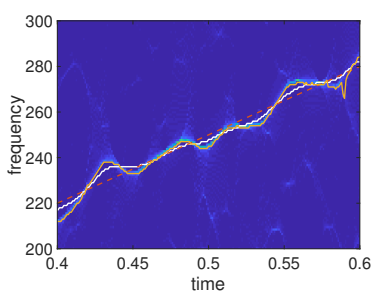

(b)
Fig. 4: (a): Modulus of FSST3 for a noisy linear chirp (SNR equal $-5 \mathrm{~dB}$ ); (b): zoom in on FSST3 modulus, on which FSST2 ridge (white plain line), FSST3 ridge (orange plain line), and the true IF (dashed line) are superimposed

modulus is displayed in Fig. 4 (a), and a zoomed in version in Fig. 4 (b), on which we superimpose the FSST2 ridge (white plain line), the FSST3 ridge (orange plain line), and true IF (dashed line), and we notice that, as expected, when the FSST2 ridge oscillates, the FFST3 ridge oscillates more, which makes the latter a less accurate IF estimator than the former.

\section{IF ESTIMATION FROM RELEVANT POINTS ON THE RIDGES OF SYNCHROSQUEEZING TRANSFORMS}

In this section, we investigate how to estimate the IF of the modes from relevant TF coefficients located on either FSST or FSST2 ridges. To extract these relevant points we first study the case of interfering pure harmonic modes and then that of noisy linear chirps. Based on that study we propose a novel algorithm for IF estimation from these relevant points. More precisely, going back to the illustration of Fig. 1, it transpires that in the case of two interfering pure harmonic modes neither the spectrogram nor FSST2 ridges are good estimates of the IF of the modes, while FSST ridges are much more accurate estimators of the IF of the modes. On the contrary, when the signal is made of modulated modes it is reported in [19] that FSST2 ridges lead to much better IF estimates than FSST ridges. So it is of interest to improve the IF estimation from FSST2 ridges for interfering pure harmonic modes in such a way that the proposed technique remains competitive on modulated modes. Note finally that, as IF estimation from FSST or FSST2 ridges is constrained by the frequency resolution, an alternative is to use $\widehat{\omega}_{f}$ (resp. $\widehat{\omega}_{f}^{[2]}$ ) on FSST (resp. FSST2) ridges as IF estimators [19], [20].

\section{A. Extraction of Relevant Points from TF Ridges of Interfering Pure Harmonic Modes}

To define relevant points on the TF ridges of interfering pure harmonic modes, we shall first remark that when $f$ is the sum of two pure harmonic modes as in Section IV, at points $\bar{t}_{k}=\frac{2 k+1}{4\left(\xi_{2}-\xi_{1}\right)}$, the spectrogram of $f$ reads

$$
\begin{aligned}
\left|V_{f}^{h}\left(\bar{t}_{k}, \eta\right)\right|^{2} & = & \sigma^{2}\left(A^{2} e^{-2 \pi \sigma^{2}\left(\eta-\xi_{1}\right)^{2}}+e^{-2 \pi \sigma^{2}\left(\eta-\xi_{2}\right)^{2}}\right) \\
& = & \left|V_{f_{1}}^{h}\left(\bar{t}_{k}, \eta\right)\right|^{2}+\left|V_{f_{2}}^{h}\left(\bar{t}_{k}, \eta\right)\right|^{2} .
\end{aligned}
$$

This means that, at these points, STFT does not see mode interference, therefore the points on the spectrogram ridge at these time instants should give the best estimates of the IFs of the modes. However, to determine the time instants $\bar{t}_{k}$ is not possible because it explicitly uses $\xi_{1}$ and $\xi_{2}$ the unknown frequencies.

At $\bar{t}_{k}$, FSST should also be sharp because $\widehat{\omega}_{f}$ is only slightly contaminated by mode interference, and this should result in local maxima of the modulus of FSST along its ridges. We are going to show that, as expected, the time instants when these local maxima occur on the ridge are good estimates of $\bar{t}_{k}$. Interestingly, these time instants $\bar{t}_{k}$ are also critical for the second order reassignment operator. Indeed, recalling that we have:

$$
\begin{aligned}
V_{f}^{t h}=\frac{i}{2 \pi} \partial_{\eta} V_{f}^{h}=-i \sigma^{3} & {\left[A\left(\eta-\xi_{1}\right) e^{-\pi \sigma^{2}\left(\eta-\xi_{1}\right)^{2}} e^{2 i \pi \xi_{1} t}\right.} \\
+ & \left.\left(\eta-\xi_{2}\right) e^{-\pi \sigma^{2}\left(\eta-\xi_{2}\right)^{2}} e^{2 i \pi \xi_{2} t}\right],
\end{aligned}
$$

one obtains that, at $t=\bar{t}_{k}$ :

$$
\left|V_{f}^{t h}\left(\bar{t}_{k}, \eta\right)\right|^{2}=\left|V_{f_{1}}^{t h}\left(\bar{t}_{k}, \eta\right)\right|^{2}+\left|V_{f_{2}}^{t h}\left(\bar{t}_{k}, \eta\right)\right|^{2} .
$$

Then the numerator of (27) is made of expressions that clearly separate the first mode from the second, and thus FSST2 should also be sharp at $\bar{t}_{k}$, resulting in significant modulus maxima (SMM) of FSST2 along its ridges. To compute these SMM we use the robust peak finder algorithm with default parameters proposed in [30]. We are going to show that the location of these SMM are actually good estimates of time instant $\bar{t}_{k}$.

To do so, we plot in Fig. 5 (a), the modulus of FSST and FSST2 along their upper ridges for the signal of Fig. 1, and first notice that their SMM are very close in time. To check that the time location of SMM are estimates of $\bar{t}_{k}$, we plot in Fig. 5 (b) and (c), STFT, FSST and FSST2 lower and upper ridges respectively, as well as the locations of SMM and also $\left(\bar{t}_{k}, \xi_{1}\right)$ or $\left(\bar{t}_{k}, \xi_{2}\right)$ depending on the cases. We observe that the SMM of FSST and FSST2 along their ridges are actually at frequencies close to the IFs of the mode and at time instants close to $\bar{t}_{k}$. We also remark that the FSST ridges are at the expected IF location, while FSST2 ridges are closer to those of the spectrogram (though higher (resp. lower) for the upper (resp. lower) ridge as predicted by Proposition IV.3). 


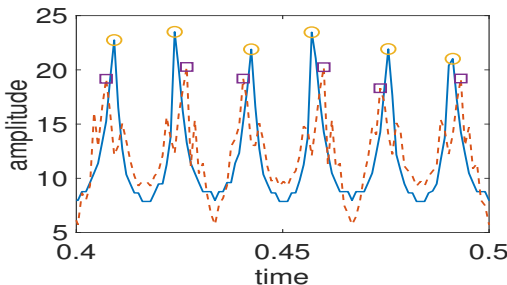

(a)

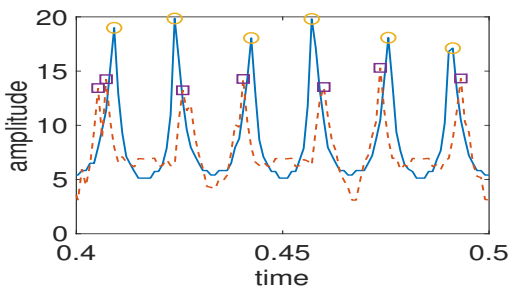

(d)

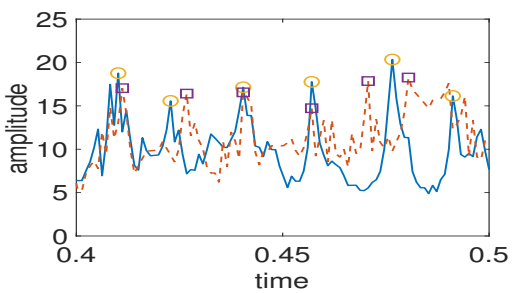

(g)

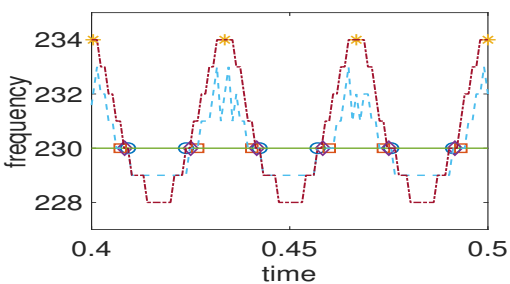

(b)

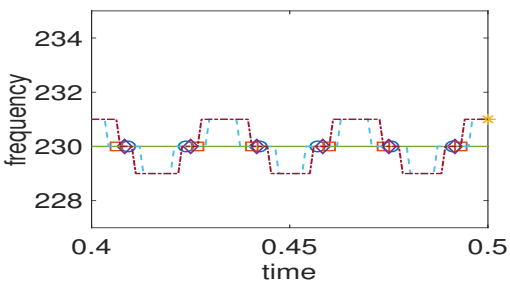

(e)

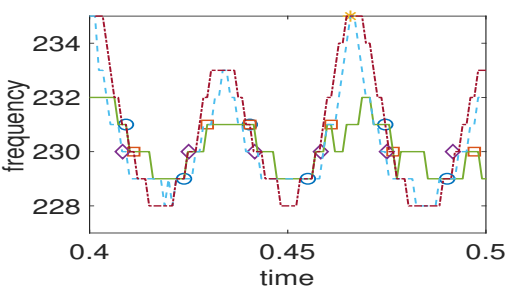

(h)

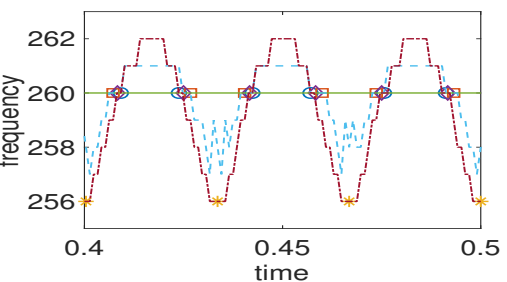

(c)

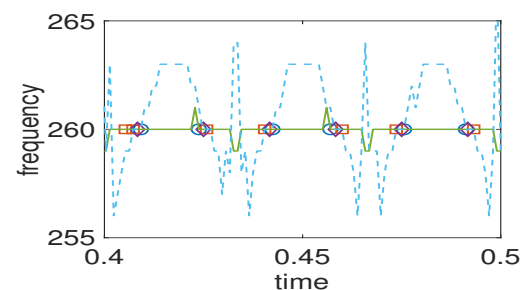

(f)

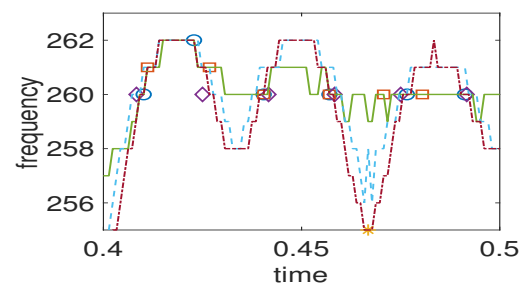

(i)

Fig. 5: (a): moduli of FSST and FSST2 along their upper ridges corresponding to TF representation of Fig. 1 (b) and (c) respectively (plain line corresponds to FSST ridge, dashed line to FSST2 ridge, circles and squares are located at SMM in each case); (b): lower STFT (dashed dotted line), FSST (plain line) and FSST2 (dashed line) ridges computed on TFRs of Fig. 1 (a) to (c) respectively (the stars, circles and squares denote the locations of SMM of each TFR along the plotted ridges, and the diamond symbols correspond to points $\left(\bar{t}_{k}, \xi_{1}\right)$ ); (c): same as (b) but for the upper ridge; (d): same computation as in (a) except the amplitude of the lower frequency mode is multiplied by 2; (e): same as (b) but for the signal used in (d); (f): same as (c) but for the signal used in (d); (g): same as (a) except some complex Gaussian white noise is added to the signal(input SNR $10 \mathrm{~dB}$ ); (h): same as (b) but for the signal considered in (g); (i): same as (c) but for the signal considered in (g).

Now, on the second row of Fig. 5, we perform the same computation except the amplitude of the lower mode is multiplied by 2 . In Fig. 5 (d), we display the moduli of FSST and FSST2 along the ridges corresponding to the upper mode. In such a case, since the amplitude of the lower mode is multiplied by 2 , the upper ridge is more impacted by interference that in the previous case. Therefore, we need to check that the SMM on these ridges are still located at frequencies close to the true IF of the modes and at time instants close to $\bar{t}_{k}$. In Fig. 5 (e) and (f), we plot STFT, FSST and FSST2 lower and upper ridges respectively, and we notice that as the amplitude of the lower mode is twice that of the upper mode, the fluctuations of the frequencies on the ridges, which are due to interference, are much less important for the lower mode that for the upper one. Interestingly, even if the FSST2 ridge corresponding to the upper mode is oscillating a lot, the significant modulus maxima on that ridge still correspond to TF points close to the IF of the mode in frequency and to $\bar{t}_{k}$ in time.

Finally, on the third row of Fig. 5 we perform the same type of computation as on the first row except we consider a noisy signal (Gaussian white noise, input SNR $=10 \mathrm{~dB}$ ).
In such an instance, the SMM along FSST and FSST2 ridges are still located at frequencies close to the IFs of the modes, the time instant corresponding to SMM being close to $\bar{t}_{k}$ in most instances. Finally, we shall mention that once the ridges are computed, to obtain an IF estimate independent of the frequency resolution one commonly uses $\widehat{\omega}_{f}\left(\right.$ resp. $\widehat{\omega}_{f}^{[2]}$ ) on FSST (resp. FSST2) ridge. In this particular example this does not lead to a significant difference in terms of IF estimation.

\section{B. Salient Points Extraction from the TF Ridge of a Noisy Linear Chirp}

We here consider that some complex Gaussian white noise $n$ is added to a linear chirp $f$ to obtain $\tilde{f}=f+n$. An important aspect is that the presence of noise creates some oscillations of the modulus of FSST and FSST2 along their corresponding ridges. As in the case of interfering pure harmonic modes, we make the hypothesis that the frequency locations of SMM along these ridges lead, on average, to a better IF estimate of the mode than the ridges themselves. To check this, we consider the linear chirp of Fig. 6 (a) and investigate IF estimation either from the FSST ridge, from SMM on the 


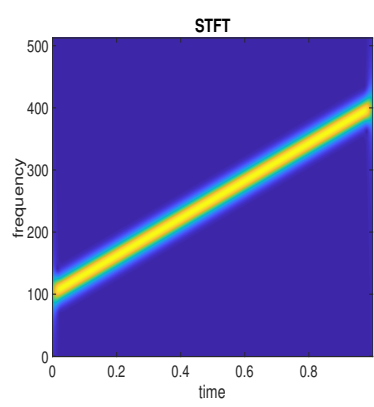

(a)

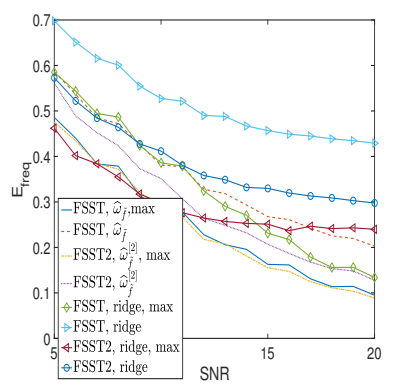

(b)
Fig. 6: (a): Example of a linear chirp; (b): Computation of $E_{\text {freq }}$ defined in (40) using different estimates computed from FSST or FSST2 ridges, at locations corresponding either to SMM on the ridges or to the whole ridges. The results are average over 40 realizations.

FSST ridge, from the FSST2 ridge or from SMM on the FSST2 ridge. We also investigate IF estimation using either $\widehat{\omega}_{\tilde{f}}$ on the FSST ridge, $\widehat{\omega}_{\tilde{f}}$ at SMM on the FSST ridge, $\widehat{\omega}_{\tilde{f}}^{[2]}$ on the FSST2 ridge or $\widehat{\omega}_{\tilde{f}}^{[2]}$ at SMM on the FSST2 ridge. To measure the quality of estimation we compute the difference between the true IF and the different estimations by means of:

$$
E_{\text {freq }}=\operatorname{mean}_{t \in I}\left|\phi^{\prime}(t)-\varphi(t)\right|,
$$

in which $\varphi$ is an estimation of $\phi^{\prime}$ and $I$ is either the whole time span or the time location of SMM.

The results depicted in Fig. 6 (b) show that better estimation results are obtained by considering only the TF locations of SMM on the ridge rather than the whole ridge, this being true for both FSST and FSST2. Then, we obtain better IF estimation by considering $\widehat{\omega}_{f}$ (resp. $\widehat{\omega}_{f}^{[2]}$ ) on the FSST (resp. FSST2) ridge rather than the FSST (resp. FSST2) ridge. Finally, to consider the values of the reassignment operators at SMM results in even better IF estimates.

\section{New IF Estimator from SMM on the Ridges of Reassigned Transforms}

In this section, we derive a new IF estimator from SMM along FSST or FSST2 ridges. We denote by $\mathcal{M}$ the set of SMM along either FSST ridges, and then consider the cubic spline $\hat{s}$ minimizing:

$$
p \sum_{(t, \eta) \in \mathcal{M}}\left|\widehat{\omega}_{\tilde{f}}(t, \eta)-s(t)\right|^{2}+(1-p) \int\left|s^{\prime \prime}(u)\right|^{2} d u,
$$

where $p$ is a trade-off parameter between the data term and the smoothing term. Note that by defining $\mathcal{M}$ from FSST2 ridges and by replacing $\widehat{\omega}_{\tilde{f}}$ by $\widehat{\omega}_{\tilde{f}}^{[2]}$, one easily adapts the optimization process (41) to FSST2 ridges.

To investigate the behavior of the proposed new IF estimator we consider the three different signals depicted in Fig. 7 (a) to (c). In each case, we add some complex Gaussian white noise and make the input SNR vary. We then display on the second row of Fig. 7, the IF estimation results measured in terms of output SNR. We first notice that the IF estimators corresponding to $\widehat{\omega}_{\tilde{f}}$ on FSST ridges or $\widehat{\omega}_{\tilde{f}}^{[2]}$ on FSST2 ridges are worse than $\hat{s}$ for the first two signals. For these signals, the best estimates are obtained with $\hat{s}$ provided some smoothing is added, and we notice that the quality of estimation does not much depend on $p$ (the results are the same for $p=0.9$ or $p=0.95$, and to take a smaller $p$ does not alter the results). For interfering pure harmonic modes $\hat{s}$ computed from FSST lead to the best results while $\hat{s}$ computed from FSST2 leads to a much better IF estimates than $\widehat{\omega}_{\tilde{f}}^{[2]}$ computed on FSST2 ridges, except if $p=1$, for which we get very similar results. For the second signal, the best estimates are obtained with $\hat{s}$ computed either from FSST or FSST2, provided some smoothing is added (the results are again the same for $p=0.9$ and $p=0.95$, and to consider a smaller $p$ does not alter the results). When $p=1$, the results are similar to those obtained with the other tested estimators. When one considers the signal of Fig. 7 (c), the situation is completely different. Indeed, the number of SMM drastically falls when FSST ridges are used: for such a signal, neither $\widehat{\omega}_{\tilde{f}}$ computed on FSST ridge nor $\hat{s}$ computed from FSST ridges are good IF estimates. On the contrary, if one considers $\hat{s}$ computed from FSST2 ridge, one again obtains similar results to those obtained with $\widehat{\omega}_{\tilde{f}}^{[2]}$ on FSST2 ridge when $p=1$, but to add some smoothing worsens the estimation.

To conclude on these experiments, we shall put the emphasis on the fact that $\hat{s}$ provides with IF estimates of the same quality as those provided by the state-of-the-art methods based on FSST or FSST2, but uses only few values of $\widehat{\omega}_{\tilde{f}}$ or $\widehat{\omega}_{\tilde{f}}^{[2]}$ on the respective ridges. To highlight this point, we compute in Fig. 8 the average proportion of points on FSST or FSST2 ridges used to compute $\hat{s}$, for the three signals on the first row of Fig. 7. We notice that this proportion increases as the noise level decreases, but remains extremely low and that, for the signal of Fig. 7 (c), FSST ridge contains much less SMM than in the other cases, which explains why the IF estimate provided by $\hat{s}$ computed from FSST ridge is a lot worse in that case.

\section{Application to a Bat Echolocation Call}

In this section we investigate the behavior of the proposed new IF estimate $\hat{s}$ on a bat echolocation call, whose STFT modulus is represented in Fig. 9 (a). For such a signal, 4 main ridges are visible, and we compute ridge detection from FSST modulus or from FSST2 modulus, assuming the number of modes is 4. The results depicted in Fig. 9 (a) and (b), show that, while ridge detection seems accurate when performed on FSST2 modulus it fails with FSST modulus. Therefore, we only carry out the computation of $\hat{s}$ from FSST2 ridges, and display the obtained IF estimators in Fig. 9 (c) (the smoothing parameter $p$ is set to 0.95 which is in accordance with the fact the fact that the bat echolocation call leads to ridge portion that are very similar to linear chirps). To better understand what is going on, we also plot the TF points $\left(t, \widehat{\omega}_{\tilde{f}}^{[2]}(t)\right)$ corresponding to SMM on FSST2 ridges (diamond symbols in Fig. 9. (c)). We notice that these SMM are located only on the most relevant portions of FSST2 ridges, and thus $\hat{s}$ is computed using only a part of FSST2 ridges. The proposed technique thus enables to extrapolate the ridges for the whole time span from SMM 


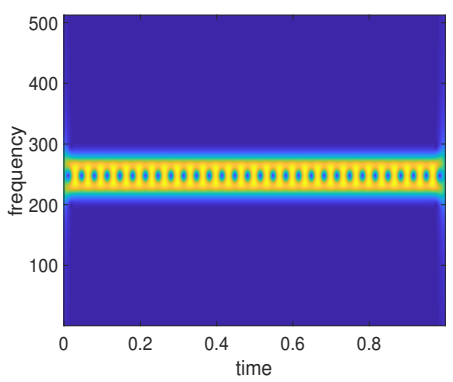

(a)

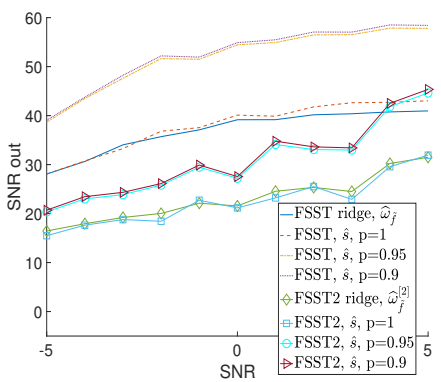

(d)

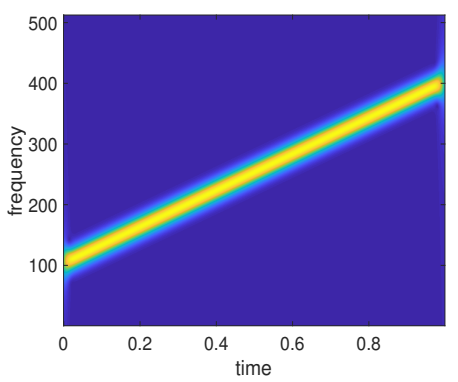

(b)

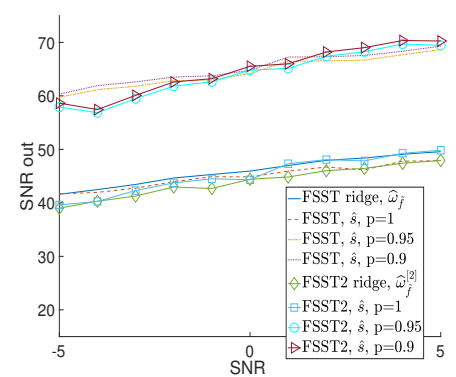

(e)

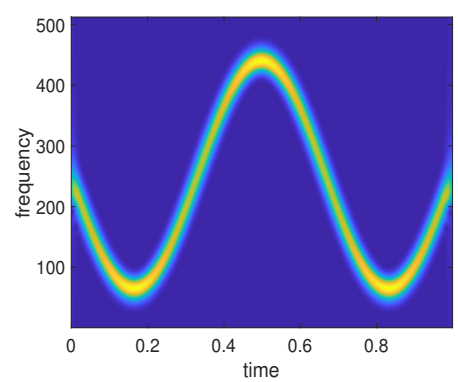

(c)

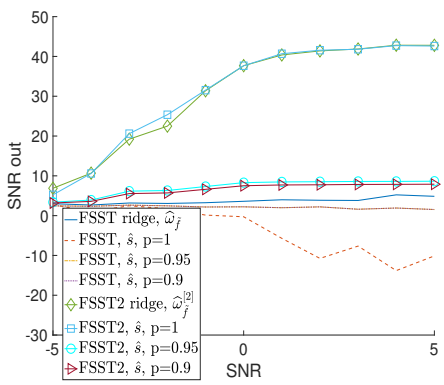

(f)

Fig. 7: (a): modulus of the STFT of two pure harmonic interfering modes; (b): modulus of the STFT of a linear chirp; (c): modulus of the STFT of a signal with cosinusoidal phase; (d): output SNR associated with IF estimations $\hat{s}$ with respect to input SNR (either computed from FSST or FSST2), $\widehat{\omega}_{f}$ on FSST ridge and $\widehat{\omega}_{f}^{[2]}$ on FSST2 ridge for noisy versions of signal (a); (e): same as (d) but for the signal displayed in (b); (f): same as (d) but for the signal displayed in (c). In (d), (e) and (f), the results are average over 40 realizations

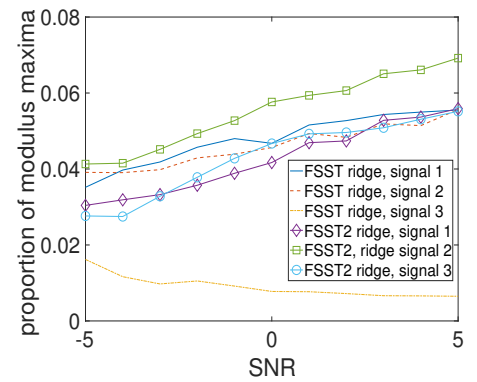

Fig. 8: Proportion of points that are SMM on FSST or FSST2 ridges, signal 1 stands for the signal of Fig. 7 (a), signal 2 for that of Fig. 7 (b) and signal 3 for that of Fig. 7 (c).

detected in regions with high energy. Nevertheless, we believe that to look for ridges lasting for the whole time span may not be meaningful in that particular example, since after time $t=0.8 \mathrm{~s}$ the energy of the signal is extremely low. So, in terms of perspectives, SMM on FSST2 ridges could also be a good indicator of the disappearance of a mode, and this direction of research will be investigated in future works.

\section{CONCLUSION}

In this paper, we proposed to analyze the behavior of the reassignment vectors used in synchrosqueezing transforms of multicomponent signals. Our goal was first to better understand the difference in behavior between these reassignment operators depending on the order of the synchrosqueezing transform. Then based on this analysis we considered two specific cases, namely interfering pure harmonic modes and noisy linear chirps. From our study, it transpired that the TF coefficients are reassigned to different locations depending on the order of the synchrosqueezing transform, and that when the latter is based on a high order polynomial approximation of the phase, interference and noise both create oscillations on the ridges corresponding to modes which drastically reduces the accuracy of IF estimation from these ridges. In a second time, we showed that by considering significant modulus maxima along the ridges of synchrosqueezing transforms, it was possible to build new IF estimators using very few TF points, whose behaviors was thoroughly analyzed both on synthetic and real signals. Potential extensions of this work will be to find a means to fully characterize the zeros of the reassignment vector of any order, to use the relevant points used in the definition of our new IF estimator to determine relevant ridge portions in the TF plane, and finally to investigate how the proposed approaches could be extended to the context of crossing modes.

\section{APPENDIX}

\section{A. Proof of Prosposition III.1}

Referring to Proposition III.1 of [21], one can write that:

$$
r_{2}^{[3]}=r_{2}^{[2]}-x_{3,2} r_{3}^{[3]} .
$$




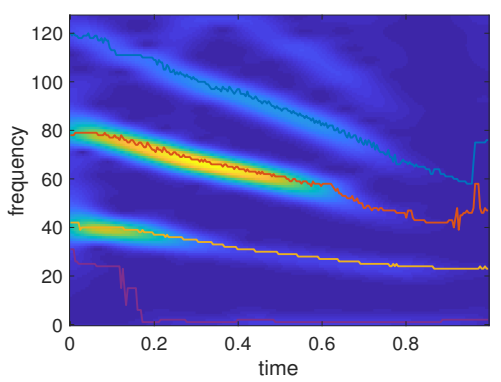

(a)

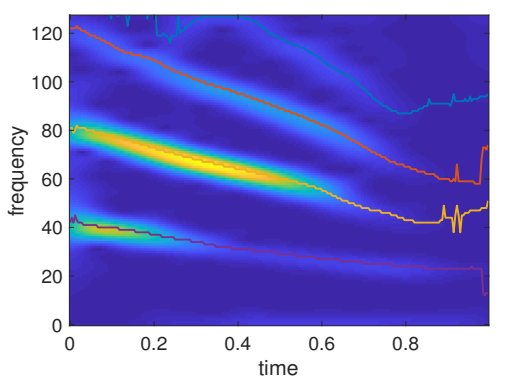

(b)

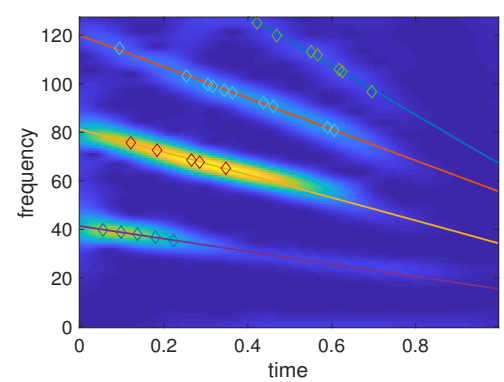

(c)

Fig. 9: (a): modulus of a bat echolocation call with FSST ridges superimposed; (b): same bat echolocation call as in (a) except the FSST2 ridges are superimposed; (c): same bat echolocation call as in (a) with $\hat{s}$ computed from FSST2 ridges superimposed, the diamond symbols are $\widehat{\omega}_{f}^{[2]}$ of the SMM selected on the FSSTs

in which $x_{k, 1}=\frac{V_{f}^{t^{k-1} h}}{2 i \pi V_{f}^{h}}$ and then $x_{k, j}=\frac{\partial_{\eta} x_{k, j-1}}{\partial_{\eta} x_{k, j-1}}$ meaning that:

$$
x_{3,2}=\frac{\partial_{\eta} x_{3,1}}{\partial_{\eta} x_{2,1}}=\frac{\partial_{\eta} \frac{V_{f}^{t^{2} h}}{V_{f}^{h}}}{\partial_{\eta} \frac{V_{f}^{t h}}{V_{f}^{h}}}=\frac{V_{f}^{t^{2} h} V_{f}^{t h}-V_{f}^{t^{3} h} V_{f}^{h}}{\left(V_{f}^{t h}\right)^{2}-V_{f}^{t^{2} h} V_{f}^{h}}
$$

As we also have:

$$
\begin{aligned}
\tilde{\omega}_{f}^{[3]} & =\tilde{\omega}_{f}-r_{2}^{[3]} x_{2,1}-r_{3}^{[3]} x_{3,1} \\
& =\tilde{\omega}_{f}^{[2]}+r_{3}^{[3]}\left(x_{2,1} x_{3,2}-x_{3,1}\right)
\end{aligned}
$$

and thus

$$
\begin{array}{r}
\widehat{\omega}_{f}^{[3]}=\widehat{\omega}_{f}^{[2]}+\Re\left\{r_{3}^{[3]}\left(x_{2,1} x_{3,2}-x_{3,1}\right)\right\} \\
=\widehat{\omega}_{f}^{[2]}+\Im\left\{\frac{r_{3}^{[3]}}{2 \pi} \frac{\left(V_{f}^{t^{2} h}\right)^{2}-V_{f}^{t^{3} h} V_{f}^{t h}}{\left(V_{f}^{t h}\right)^{2}-V_{f}^{t^{2} h} V_{f}^{h}}\right\} \\
=\widehat{\omega}_{f}^{[2]}+\Im\left\{\frac{r_{3}^{[3]}}{2 \pi}\right\} \Re\left\{\frac{\left(V_{f}^{t^{2} h}\right)^{2}-V_{f}^{t^{3} h} V_{f}^{t h}}{\left(V_{f}^{t h}\right)^{2}-V_{f}^{t^{2} h} V_{f}^{h}}\right\} \\
+\Re\left\{\frac{r_{3}^{[3]}}{2 \pi}\right\} \Im\left\{\frac{\left(V_{f}^{t^{2} h}\right)^{2}-V_{f}^{t^{3} h} V_{f}^{t h}}{\left(V_{f}^{t h}\right)^{2}-V_{f}^{t^{2} h} V_{f}^{h}}\right\} \\
=\widehat{\omega}_{f}^{[2]}+\Im\left\{\frac{r_{3}^{[3]}}{2 \pi}\right\} \Re\left\{\frac{\left(V_{f}^{t^{2} h}\right)^{2}-V_{f}^{t^{3} h} V_{f}^{t h}}{\left(V_{f}^{t h}\right)^{2}-V_{f}^{t^{2} h} V_{f}^{h}}\right\} .
\end{array}
$$

Note that the last equality holds because $\log (A)$ is a second order polynomial.

\section{B. Proof of Proposition IV.1}

In the context of Proposition IV.1, the zeros of $\widehat{\omega}_{f}-\eta$ satisfy:

$$
\begin{array}{r}
\partial_{\eta}\left|V_{f}^{h}(t, \eta)\right|^{2}=0 \\
\Leftrightarrow A^{2}\left(\eta-\xi_{1}\right) e^{-2 \pi \sigma^{2}\left(\eta-\xi_{1}\right)^{2}}+\left(\eta-\xi_{2}\right) e^{-2 \pi \sigma^{2}\left(\eta-\xi_{2}\right)^{2}}+ \\
A\left(2 \eta-\xi_{1}-\xi_{2}\right) e^{-\pi \sigma^{2}\left[\left(\eta-\xi_{1}\right)^{2}+\left(\eta-\xi_{2}\right)^{2}\right]} \cos \left(2 \pi\left(\xi_{2}-\xi_{1}\right) t\right)=0 .
\end{array}
$$

Then, among these zeros, those satisfying $V_{f}^{h} V_{f}^{t^{2} h}-\left(V_{f}^{t h}\right)^{2} \neq$ 0 are also zeros of $\widehat{\omega}_{f}^{[2]}-\eta$ if they satisfy:

$$
\begin{array}{r}
\partial_{\eta}\left|V_{f}^{t h}(t, \eta)\right|^{2}=\frac{1}{4 \pi^{2}} \partial_{\eta}\left|\partial_{\eta} V_{f}^{h}(t, \eta)\right|^{2} \\
=\sigma^{6}\left(2 \partial_{\eta}\left|V_{f}^{h}(t, \eta)\right|^{2}-A^{2} 4 \pi \sigma^{2}\left(\eta-\xi_{1}\right)^{3} e^{-2 \pi \sigma^{2}\left(\eta-\xi_{1}\right)^{2}}\right. \\
-4 \pi \sigma^{2}\left(\eta-\xi_{2}\right)^{3} e^{-2 \pi \sigma^{2}\left(\eta-\xi_{2}\right)^{2}}-4 A \pi \sigma^{2}\left(\eta-\xi_{1}\right)\left(\eta-\xi_{2}\right) \\
\left.\left(2 \eta-\xi_{1}-\xi_{2}\right) e^{-\pi \sigma^{2}\left[\left(\eta-\xi_{1}\right)^{2}+\left(\eta-\xi_{2}\right)^{2}\right]} \cos \left(2 \pi\left(\xi_{2}-\xi_{1}\right) t\right)\right)=0 .
\end{array}
$$

Then, from these two equations we get that the zeros of $\widehat{\omega}_{f}-\eta$ are zeros of $\widehat{\omega}_{f}^{[2]}-\eta$ if and only if:

$$
\begin{array}{r}
-A^{2}\left(\eta-\xi_{1}\right)^{3} e^{-2 \pi \sigma^{2}\left(\eta-\xi_{1}\right)^{2}}-\left(\eta-\xi_{2}\right)^{3} e^{-2 \pi \sigma^{2}\left(\eta-\xi_{2}\right)^{2}} \\
-A\left(\eta-\xi_{1}\right)\left(\eta-\xi_{2}\right)\left(2 \eta-\xi_{1}-\xi_{2}\right) e^{-\pi \sigma^{2}\left[\left(\eta-\xi_{1}\right)^{2}+\left(\eta-\xi_{2}\right)^{2}\right]} \\
\left.\cos \left(2 \pi\left(\xi_{2}-\xi_{1}\right) t\right)\right) \\
=\left(\xi_{2}-\xi_{1}\right) \\
{\left[-A^{2}\left(\eta-\xi_{1}\right)^{2} e^{-2 \pi \sigma^{2}\left(\eta-\xi_{1}\right)^{2}}+\left(\eta-\xi_{2}\right)^{2} e^{-2 \pi \sigma^{2}\left(\eta-\xi_{2}\right)^{2}}\right]=0 .}
\end{array}
$$

or equivalently:

$$
A\left(\eta-\xi_{1}\right) e^{-\pi \sigma^{2}\left(\eta-\xi_{1}\right)^{2}}= \pm\left(\eta-\xi_{2}\right) e^{-\pi \sigma^{2}\left(\eta-\xi_{2}\right)^{2}} .
$$

\section{Proof of Proposition IV.2}

Assume $A\left(\eta-\xi_{1}\right) e^{-\pi \sigma^{2}\left(\eta-\xi_{1}\right)^{2}}=-\left(\eta-\xi_{2}\right) e^{-\pi \sigma^{2}\left(\eta-\xi_{2}\right)^{2}}$, then we may write:

$$
\begin{array}{r}
\partial_{\eta}\left|V_{f}^{h}(t, \eta)\right|^{2}=0 \Leftrightarrow \\
\left(2 \eta-\xi_{1}-\xi_{2}\right)\left(\eta-\xi_{1}\right) \\
\left(1-\cos \left(2 \pi\left(\xi_{2}-\xi_{1}\right) t\right)\right) e^{-2 \pi \sigma^{2}\left(\eta-\xi_{2}\right)^{2}}=0 \\
\Leftrightarrow t=t_{k}=\frac{k}{\xi_{2}-\xi_{1}},
\end{array}
$$

since it can be easily shown that the $\eta$ of interest are different from $\xi_{1}$ and $\frac{\xi_{1}+\xi_{2}}{2}$. When $A\left(\eta-\xi_{1}\right) e^{-\pi \sigma^{2}\left(\eta-\xi_{1}\right)^{2}}=(\eta-$ $\left.\xi_{2}\right) e^{-\pi \sigma^{2}\left(\eta-\xi_{2}\right)^{2}}$, we similarly obtain that the time instants $t$ corresponding to the studied zeros are the $\tilde{t}_{k}=\frac{k+1 / 2}{\xi_{2}-\xi_{1}}, k \in \mathbb{Z}$.

\section{Proof of Proposition IV.3}

As one can easily show that $\eta_{2}^{\max }<\eta_{2}^{\min }$, and as $\left|V_{f}^{h}\left(t_{k}, .\right)\right|^{2}$ attains a maximum at $\eta_{2}^{\max }$ its derivative is 
negative on $\left[\eta_{2}^{\max }, \eta_{2}^{\min }\right]$, which is, using (34), equivalent to:

$$
-A\left(\eta-\xi_{1}\right) e^{-\pi \sigma^{2}\left(\eta-\xi_{1}\right)^{2}} \leq\left(\eta-\xi_{2}\right) e^{-\pi \sigma^{2}\left(\eta-\xi_{2}\right)^{2}} .
$$

Similarly, remarking that $\left|V_{f}^{h}\left(\tilde{t}_{k}, .\right)\right|^{2}$ attains a maximum at $\eta_{2}^{\min }$, its derivative is positive on $\left[\eta_{2}^{\max }, \eta_{2}^{\min }\right]$, meaning that, on that interval

$$
-A\left(\eta-\xi_{1}\right) e^{-\pi \sigma^{2}\left(\eta-\xi_{1}\right)^{2}} \geq-\left(\eta-\xi_{2}\right) e^{-\pi \sigma^{2}\left(\eta-\xi_{2}\right)^{2}}
$$

Putting (45) and (46) together, we get that on the interval $\left[\eta_{2}^{\max }, \eta_{2}^{\min }\right]$ :

$$
\left|A\left(\eta-\xi_{1}\right) e^{-\pi \sigma^{2}\left(\eta-\xi_{1}\right)^{2}}\right| \leq\left|\left(\eta-\xi_{2}\right) e^{-\pi \sigma^{2}\left(\eta-\xi_{2}\right)^{2}}\right| .
$$

Then, we remark that on the upper spectrogram ridge, $\eta$ belongs to $\left[\eta_{2}^{\max }, \eta_{2}^{\min }\right]$. Using arguments developed in the proof of Proposition IV.1, one can write that at such $(t, \eta)$, i.e. such that $\partial_{\eta}\left|V_{f}^{h}(t, \eta)\right|^{2}=0$, one has:

$$
\begin{array}{r}
\widehat{\omega}_{f}^{[2]}(t, \eta)-\eta \\
=\frac{\left|V_{f}^{h}(t, \eta)\right|^{2} \partial_{\eta}\left|V_{f}^{t h}(t, \eta)\right|^{2}}{\left|V_{f}^{h}(t, \eta) V_{f}^{t^{2} h}(t, \eta)-\left(V_{f}^{t h}(t, \eta)\right)^{2}\right|^{2}} \\
=4 \pi \sigma^{8} \frac{\left|V_{f}^{h}(t, \eta)\right|^{2}\left(\xi_{2}-\xi_{1}\right)}{\left|V_{f}^{h}(t, \eta) V_{f}^{t^{2} h}(t, \eta)-\left(V_{f}^{t h}(t, \eta)\right)^{2}\right|^{2}} \\
{\left[-A^{2}\left(\eta-\xi_{1}\right)^{2} e^{-2 \pi \sigma^{2}\left(\eta-\xi_{1}\right)^{2}}+\left(\eta-\xi_{2}\right)^{2} e^{-2 \pi \sigma^{2}\left(\eta-\xi_{2}\right)^{2}}\right],}
\end{array}
$$

which has the sign of $-A^{2}\left(\eta-\xi_{1}\right)^{2} e^{-2 \pi \sigma^{2}\left(\eta-\xi_{1}\right)^{2}}+(\eta-$ $\left.\xi_{2}\right)^{2} e^{-2 \pi \sigma^{2}\left(\eta-\xi_{2}\right)^{2}}$. Using (47), we can thus deduce that the reassignment vector on the upper spectrogram ridge is oriented towards higher frequencies and is null at points $\left(\tilde{t}_{k}, \eta_{2}^{\min }\right)$ and $\left(t_{k}, \eta_{2}^{\max }\right)$.

The same reasoning can be carried out for the lower ridge, namely studying the reassignment vector on the interval $\left[\eta_{0}^{\min }, \eta_{0}^{\max }\right]$, and enables us to show that the second order reassignment vector on that ridge is oriented towards lower frequencies.

\section{REFERENCES}

[1] U. R. Acharya, K. P. Joseph, N. Kannathal, L. C. Min, and J. S. Suri, "Heart rate variability," in Advances in cardiac signal processing. Springer, 2007, pp. 121-165.

[2] M. Malik and A. J. Camm, Dynamic electrocardiography. John Wiley \& Sons, 2008

[3] M. Costa, A. A. Priplata, L. A. Lipsitz, Z. Wu, N. E. Huang, A. L. Goldberger, and C.-K. Peng, "Noise and poise: Enhancement of postural complexity in the elderly with a stochastic-resonance-based therapy," Europhysics Letters (EPL), vol. 77, no. 6, p. 68008, Mar 2007.

[4] D. A. Cummings, R. A. Irizarry, N. E. Huang, T. P. Endy, A. Nisalak, K. Ungchusak, and D. S. Burke, "Travelling waves in the occurrence of dengue haemorrhagic fever in Thailand," Nature, vol. 427, no. 6972, pp. $344-347$, Jan 2004.

[5] C. L. Herry, M. Frasch, A. J. Seely, and H.-T. Wu, "Heart beat classification from single-lead ECG using the synchrosqueezing transform," Physiological Measurement, vol. 38, no. 2, pp. 171-187, 2017.

[6] P. Flandrin, Time-frequency/time-scale analysis. Academic Press, 1998, vol. 10.

[7] L. Stankovic, M. Dakovic, and V. Ivanovic, "Performance of spectrogram as IF estimator," Electronics Letters, vol. 37, no. 12, pp. 797-799, 2001.

[8] R. Carmona, W. Hwang, and B. Torresani, "Multiridge detection and time-frequency reconstruction," IEEE Transactions on Signal Processing, vol. 47, no. 2, pp. 480-492, Feb 1999.
[9] T. Oberlin, S. Meignen, and V. Perrier, "The Fourier-based synchrosqueezing transform," in 2014 IEEE International Conference on Acoustics, Speech and Signal Processing (ICASSP), May 2014, pp. 315319.

[10] G. Thakur and H.-T. Wu, "Synchrosqueezing-based recovery of instantaneous frequency from nonuniform samples." SIAM J. Math. Analysis, vol. 43, no. 5, pp. 2078-2095, 2011.

[11] I. Daubechies and S. Maes, "A nonlinear squeezing of the continuous wavelet transform based on auditory nerve models," Wavelets in medicine and biology, pp. 527-546, 1996.

[12] I. Daubechies, J. Lu, and H.-T. Wu, "Synchrosqueezed wavelet transforms: an empirical mode decomposition-like tool," Applied and Computational Harmonic Analysis, vol. 30, no. 2, pp. 243-261, 2011.

[13] G. Yu, T. Lin, Z. Wang, and Y. Li, "Time-reassigned multisynchrosqueezing transform for bearing fault diagnosis of rotating machinery," IEEE Transactions on Industrial Electronics, vol. 68, no. 2, pp 1486-1496, 2020.

[14] C. Li and M. Liang, "Time-frequency signal analysis for gearbox fault diagnosis using a generalized synchrosqueezing transform," Mechanical Systems and Signal Processing, vol. 26, pp. 205-217, 2012.

[15] X. Wang, B. Wang, and W. Chen, "The second-order synchrosqueezing continuous wavelet transform and its application in the high-speed-train induced seismic signal," IEEE Geoscience and Remote Sensing Letters, 2020.

[16] H.-T. Wu, Y.-H. Chan, Y.-T. Lin, and Y.-H. Yeh, "Using synchrosqueezing transform to discover breathing dynamics from ecg signals," Applied and Computational Harmonic Analysis, vol. 36, no. 2, pp. 354-359, 2014.

[17] H.-t. Wu, R. Talmon, and Y.-L. Lo, "Assess sleep stage by modern signal processing techniques," IEEE Transactions on Biomedical Engineering, vol. 62 , no. 4, pp. 1159-1168, 2014.

[18] J. M. Miramont, M. A. Colominas, and G. Schlotthauer, "Voice jitter estimation using high-order synchrosqueezing operators," IEEE/ACM Transactions on Audio, Speech, and Language Processing, 2020.

[19] R. Behera, S. Meignen, and T. Oberlin, "Theoretical analysis of the second-order synchrosqueezing transform," Applied and Computational Harmonic Analysis, vol. 45, no. 2, pp. 379-404, 2018.

[20] T. Oberlin, S. Meignen, and V. Perrier, "Second-order synchrosqueezing transform or invertible reassignment? Towards ideal time-frequency representations," IEEE Transactions on Signal Processing, vol. 63, no. 5, pp. 1335-1344, March 2015.

[21] D. H. Pham and S. Meignen, "High-order synchrosqueezing transform for multicomponent signals analysis-with an application to gravitationalwave signal." IEEE Trans. Signal Processing, vol. 65, no. 12, pp. 31683178, 2017.

[22] X. Zhu, Z. Zhang, J. Gao, and W. Li, "Two robust approaches to multicomponent signal reconstruction from STFT ridges," Mechanical Systems and Signal Processing, vol. 115, pp. 720-735, 2019.

[23] L. Li, H. Cai, and Q. Jiang, "Adaptive synchrosqueezing transform with a time-varying parameter for non-stationary signal separation," Applied and Computational Harmonic Analysis, 2019.

[24] L. Li, H. Cai, H. Han, Q. Jiang, and H. Ji, "Adaptive short-time Fourier transform and synchrosqueezing transform for non-stationary signal separation," Signal Processing, vol. 166, p. 107231, 2020.

[25] V. Bruni, M. Tartaglione, and D. Vitulano, "On the time-frequency reassignment of interfering modes in multicomponent FM signals," in 2018 26th European Signal Processing Conference (EUSIPCO). IEEE, 2018, pp. 722-726.

[26] — "A fast and robust spectrogram reassignment method," Mathematics, vol. 7, no. 4, p. 358, 2019.

[27] R. G. Baraniuk, P. Flandrin, A. J. Janssen, and O. J. Michel, "Measuring time-frequency information content using the Rényi entropies," IEEE Transactions on Information theory, vol. 47, no. 4, pp. 1391-1409, 2001.

[28] A. Berrian and N. Saito, "Adaptive synchrosqueezing based on a quilted short-time fourier transform," in Wavelets and Sparsity XVII, vol. 10394. International Society for Optics and Photonics, 2017, p. 1039420.

[29] S. Meignen, M. Colominas, and D.-H. Pham, "On the use of Rényi entropy for optimal window size computation in the short-time Fourier transform," in ICASSP 2020-2020 IEEE International Conference on Acoustics, Speech and Signal Processing (ICASSP). IEEE, 2020, pp. 5830-5834.

[30] N. Yoder, "Peakfinder," Internet: http://www. mathworks. com/matlabcentral/fileexchange/25500, 2011. 


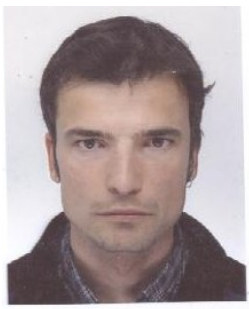

Sylvain Meignen received the Ph.D degree in applied mathematics in 2001, and its "habilitation à diriger des recherches" in 2011, both from the university of Grenoble, France. Since, 2002, he has been an associate professor at Grenoble institute of technology. His research interests include nonlinear multiscale image and signal processing, time-frequency analysis (empirical mode decomposition,synchrosqueezing) and approximation theory. In 2010, he was a visitor at the GIPSA-Lab Grenoble, at the IDCOM Laboratory of the university of Edinburgh, U.K., in 2011, and at the university of Pisa, Italy, in 2018.

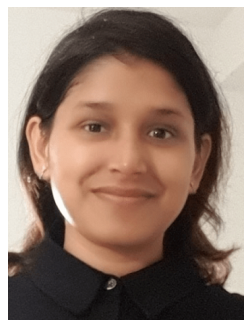

Neha Singh received the Ph.D. degree in Electronics and Communication Engineering from Indian Institute of Technology, Roorkee, India in 2019 and M.Tech degree in Robotics from Indian Institute of Information Technology, Allahabad, India in 2014. She worked as a post-doctoral researcher at LJK Laboratory in University of Grenoble, Alpes, France in 2020-2021. Her research interests include signal processing, machine learning, time-frequency analysis (synchrosqueezing and reassignment). 